\title{
HAMILTONIAN-PRESERVING SCHEMES FOR THE LIOUVILLE EQUATION WITH DISCONTINUOUS POTENTIALS*
}

\author{
SHI JIN $^{\dagger}$ AND XIN WEN ${ }^{\ddagger}$
}

\begin{abstract}
When numerically solving the Liouville equation with a discontinuous potential, one faces the problem of selecting a unique, physically relevant solution across the potential barrier, and the problem of a severe time step constraint due to the CFL condition. In this paper, we introduce two classes of Hamiltonian-preserving schemes for such problems. By using the constant Hamiltonian across the potential barrier, we introduce a selection criterion for a unique, physically relevant solution to the underlying linear hyperbolic equation with singular coefficients. These schemes have a hyperbolic CFL condition, which is a significant improvement over a conventional discretization. These schemes are proved to be positive, and stable in both $l^{\infty}$ and $l^{1}$ norms. Numerical experiments are conducted to study the numerical accuracy.

This work is motivated by the well-balanced kinetic schemes by Perthame and Simeoni for the shallow water equations with a discontinuous bottom topography, and has applications to the level set methods for the computations of multivalued physical observables in the semiclassical limit of the linear Schrödinger equation with a discontinuous potential, among other applications.
\end{abstract} limit

Key words. Liouville equation, discontinuous potential, Hamiltonian-preserving, semiclassical

AMS subject classifications. 35L45, 65M06, 70H99

\section{Introduction}

In this paper, we construct and study a class of numerical schemes for the $d$ dimensional Liouville equation in classical mechanics:

$$
f_{t}+\mathbf{v} \cdot \nabla_{\mathbf{x}} f-\nabla_{\mathbf{x}} V \cdot \nabla_{\mathbf{v}} f=0, \quad t>0, \quad \mathbf{x}, \mathbf{v} \in R^{d},
$$

where $f(t, \mathbf{x}, \mathbf{v})$ is the density distribution of a classical particle at position $\mathbf{x}$, time $t$ and travelling with velocity $\mathbf{v} . V(\mathbf{x})$ is the potential. The Liouville equation is a different formulation of Newton's second law:

$$
\frac{d \mathbf{x}}{d t}=\mathbf{v}, \quad \frac{d \mathbf{v}}{d t}=-\nabla_{\mathbf{x}} V
$$

which is a Hamiltonian system with the Hamiltonian

$$
H=\frac{1}{2}|\mathbf{v}|^{2}+V(\mathbf{x})
$$

It is known from classical mechanics that the Hamiltonian remains constant across a potential barrier.

The Liouville equation is a linear wave equation, with the characteristic speed determined by the Newton's equation (1.2)-which is usually called the bicharacteristic. If $V(\mathbf{x})$ is smooth, then the initial value problem to (1.2) is well-posed, and

${ }^{*}$ Received: January 25, 2005; accepted (in revised version); May 17, 2005. Communicated by Lenya Ryzhik.

Research supported in part by NSF grant No. DMS-0305080, NSFC under the Project 10228101 and the Basic Research Projects of Tsinghua University under the Project JC2002010.

${ }^{\dagger}$ Department of Mathematics, University of Wisconsin, Madison, WI 53706, USA, and Department of Mathematical Sciences, Tsinghua University, Beijing 100084, P.R. China (jin@math. wisc.edu).

${ }^{\ddagger}$ Department of Mathematical Sciences, Tsinghua University, Beijing 100084, P.R. China (wenxin @amss.ac.cn). 
a standard numerical method (for example, the upwind scheme and its higher order extensions) for linear wave equations give satisfactory results. However, if $V(\mathbf{x})$ is discontinuous corresponding to a potential barrier then the characteristic speed of the Liouville equation given by (1.2) is infinity at the discontinuous point. When numerically approximating $V_{x}$ across the interface, the numerical derivative of $V$ is of $O(1 / \Delta x)$, with $\Delta x$ the mesh size in the physical space. Thus an explicit scheme needs time step $\Delta t=O(\Delta x \Delta \xi)$ with $\Delta \xi$ the mesh size in particle velocity space. This is very expensive. Moreover, a conventional numerical scheme in general does not preserve a constant Hamiltonian across the interface, and usually leads to poor or even incorrect numerical resolutions by ignoring the discontinuities of $V(x)$. Theoretically, there is a uniqueness issue for weak solutions to these linear hyperbolic equations with singular wave speeds $[2,4,7,22,23]$. It is not clear which weak solution a standard numerical discretization that ignores the discontinuity of $V(x)$ will select.

Potential barriers appear in many important physical problems, such as the quantum tunnelling (and quantum dots) in semiconductor device modelling, plasmas, and geometrical optics through different materials. Liouville or Vlasov equations describe the density distribution of particles in such a heterogeneous medium. For some recent mathematical studies of discontinuous potentials in high frequency waves see $[1,19,24]$.

In this paper, we construct a class of numerical schemes that are suitable for the Liouville equation (1.1) with a discontinuous potential. An important feature of these schemes is that they are consistent to the constant Hamiltonian across a potential barrier for the Liouville equation (1.1). We call such schemes Hamiltonian-preserving schemes. A key idea in this paper is to use the behavior of a classical particle at the potential barrier-either to cross over if its kinetic energy is sufficiently large- or to be reflected with a velocity in the opposite direction. We build this mechanism into the numerical scheme to construct the Hamiltonian-preserving schemes. This work is motivated by the well-balanced kinetic scheme of Perthame and Simeoni [21] for the shallow water equations with a (discontinuous) bottom topography, in which the same mechanism was built into a hydrodynamic scheme for the shallow water equations in order to capture the steady state solutions-corresponding to a constant energy- of the shallow water equations. However, the work of Perthame and Simeoni was focused on a kinetic scheme for the shallow water equations defined in the physical space, thus the numerical discretization in the phase space was not studied. The phase space discretization is an important issue for the Liouville equation with a discontinuous potential. As shown by this work, if designed properly, the explicit Hamiltonian-preserving schemes allow a standard hyperbolic CFL condition $\Delta t=O(\Delta x, \Delta \xi)$. More importantly, by using the a constant Hamiltonian condition across the potential barrier, these schemes select a unique, and physically relevant, solution for the underlying linear hyperbolic equation with singular coefficients.

Another application of the Liouville equation like (1.1) is the level set method for the computation of multivalued solutions to quasilinear PDEs, see [12, 3]. Such problems arise in the semiclassical limit of the linear Schrödinger equation, which yields the Liouville equation (1.1) with the initial data

$$
f(\mathbf{x}, \mathbf{v}, 0)=\rho_{0}(\mathbf{x}) \delta\left(\mathbf{v}-\mathbf{u}_{0}(\mathbf{x})\right),
$$

see for example $[18,9]$. In the physical space, the moments of $f$ :

$$
\rho(\mathbf{x}, t)=\int f(\mathbf{x}, \mathbf{v}, t) d \mathbf{v},
$$




$$
\mathbf{u}(\mathbf{x}, t)=\frac{1}{\rho(\mathbf{x}, t)} \int f(\mathbf{x}, \mathbf{v}, t) \mathbf{v} d \mathbf{v}
$$

may become multivalued, see $[10,27]$ and the relevant numerical methods $[8,6]$. The level set method proposed in [11] solves the Liouville equation (1.1) with the initial data (1.4) by decomposing $f$ into $\phi$ and $\psi_{i}(i=1, \cdots, d)$ where $\phi$ and $\psi_{i}$ solve the same Liouville equation (1.1) with initial data

$$
\phi(\mathbf{x}, \mathbf{v}, 0)=\rho_{0}(\mathbf{x}), \quad \psi_{i}(\mathbf{x}, \mathbf{v}, 0)=v_{i}-u_{i 0}(\mathbf{x}),
$$

respectively. This allows the numerical computations for a bounded solution rather than a measure-valued solution of the Liouville equation with singular initial data (1.4), which greatly enhances the numerical resolution. The moments can be recovered through

$$
\begin{aligned}
& \rho(\mathbf{x}, t)=\int \phi(\mathbf{x}, \mathbf{v}, t) \Pi_{i=1}^{d} \delta\left(\psi_{i}\right) d \mathbf{v} \\
& \mathbf{u}(\mathbf{x}, t)=\int \phi(\mathbf{x}, \mathbf{v}, t) \mathbf{v} \Pi_{i=1}^{d} \delta\left(\psi_{i}\right) d \mathbf{v} / \rho(\mathbf{x}, t) .
\end{aligned}
$$

Numerical computations of a multivalued solution using this technique were given in [11] for smooth potentials. In this article we will also apply the Hamiltonianpreserving schemes for the level set computations of a multivalued solution of the physical observables $\rho, \mathbf{u}$, etc.

In Sections 2, we first point out the problems with the usual finite difference scheme to solve the Liouville equation with discontinuous potentials. We then present the designing principle of our Hamiltonian-preserving schemes using the behavior of classical particles at a potential barrier. In Section 3, a 1d Hamiltonian-preserving scheme based on a finite difference approach (called Scheme I) is given, and its positivity and $l^{\infty}$ are established. We also state our $l^{1}$ stability results, which are proved in a separate paper [13]. In Section 4, a 1D Hamiltonian-preserving scheme based on a finite volume approach (called Scheme II) is given. In Section 5, we prove that Scheme II is positive, $l^{\infty}$-stable, and $l^{1}$-contracting (for more general $l^{1}$ initial data than Scheme I). We extend these schemes to two space dimension in Section 6. In Section 7, we show that even for smooth initial data, the solution to the Liouville equation (1.1) could become discontinuous in the downstream part of the potential barrier. This contributes to the reduced numerical convergence rate to $1 / 2$ for a formally first order scheme, as in any shock capturing method for a linear wave equation with discontinuous initial data. Numerical examples are given in Section 8 to verify the accuracy of the two schemes. We conclude the paper in Section 9.

\section{The designing principle of the Hamiltonian-preserving schemes}

2.1. Deficiency of the usual finite difference schemes. We consider the numerical solution of the 1D Liouville equation

$$
f_{t}+\xi f_{x}-V_{x} f_{\xi}=0
$$

with a discontinuous potential $V(x)$.

Without loss of generality, we employ an uniform mesh with grid points at $x_{i+\frac{1}{2}}, i=0, \cdots, N$, in the $x$-direction and $\xi_{j+\frac{1}{2}}, j=0, \cdots, M$ in the $\xi$-direction. The cells are centered at $\left(x_{i}, \xi_{j}\right), i=1, \cdots, N, j=1, \cdots, M$ with $x_{i}=\frac{1}{2}\left(x_{i+\frac{1}{2}}+x_{i-\frac{1}{2}}\right)$ and 
$\xi_{j}=\frac{1}{2}\left(\xi_{j+\frac{1}{2}}+\xi_{j-\frac{1}{2}}\right)$. The mesh size is denoted by $\Delta x=x_{i+\frac{1}{2}}-x_{i-\frac{1}{2}}, \Delta \xi=\xi_{j+\frac{1}{2}}-\xi_{j-\frac{1}{2}}$. We also assume a uniform time step $\Delta t$ and the discrete time is given by $0=t_{0}<t_{1}<$ $\cdots<t_{L}=T$. We introduce mesh ratios $\lambda_{x}^{t}=\frac{\Delta t}{\Delta x}, \lambda_{\xi}^{t}=\frac{\Delta t}{\Delta \xi}, \lambda_{x}^{\xi}=\frac{\Delta \xi}{\Delta x}$, assumed to be fixed. We define the cell averages of $f$ as

$$
f_{i j}=\frac{1}{\Delta x \Delta \xi} \int_{x_{i-\frac{1}{2}}}^{x_{i+\frac{1}{2}}} \int_{\xi_{j-\frac{1}{2}}}^{\xi_{j+\frac{1}{2}}} f(x, \xi, t) d \xi d x
$$

The 1-d average quantity $f_{i+1 / 2, j}$ is defined as

$$
f_{i+1 / 2, j}=\frac{1}{\Delta \xi} \int_{\xi_{j-\frac{1}{2}}}^{\xi_{j+\frac{1}{2}}} f\left(x_{i+1 / 2}, \xi, t\right) d \xi .
$$

$f_{1, j+1 / 2}$ is defined similarly.

A typical semi-discrete finite difference method for this equation is

$$
\partial_{t} f_{i j}+\xi_{j} \frac{f_{i+\frac{1}{2}, j}-f_{i-\frac{1}{2}, j}}{\Delta x}-D V_{i} \frac{f_{i, j+\frac{1}{2}}-f_{i, j-\frac{1}{2}}}{\Delta \xi}=0,
$$

where the numerical fluxes $f_{i+\frac{1}{2}, j}, f_{i, j+\frac{1}{2}}$ are defined by the upwind scheme, and $D V_{i}$ is some numerical approximation of $V_{x}$ at $x=x_{i}$.

Such a discretization suffers from at least two problems:

- The above discretization in general does not preserve a constant Hamiltonian $H=\frac{1}{2} \xi^{2}+V$ across the discontinuities of $V$. Such a numerical approximation may lead to an unphysical solution or poor numerical resolution.

- If an explicit time discretization is used, the CFL condition for this scheme requires the time step to satisfy

$$
\Delta t\left[\frac{\max _{j}\left|\xi_{j}\right|}{\Delta x}+\frac{\max _{i}\left|D V_{i}\right|}{\Delta \xi}\right] \leq 1 .
$$

Since the potential $V(x)$ is discontinuous at some points, $\max _{i}\left|D V_{i}\right|=$ $O(1 / \Delta x)$, so the CFL condition (2.3) requires $\Delta t=O(\Delta x \Delta \xi)$.

2.2. Behavior of a classical particle at a potential barrier. In classical mechanics, a particle will either cross a potential barrier with a changing momentum, or be reflected, depending on its momentum and on the strength of the potential barrier. The Hamiltonian $H=\frac{1}{2} \xi^{2}+V$ should be preserved across the potential barrier:

$$
\frac{1}{2}\left(\xi^{+}\right)^{2}+V^{+}=\frac{1}{2}\left(\xi^{-}\right)^{2}+V^{-}
$$

where the superscripts \pm indicate the right and left limits of the quantity at the potential barrier.

For example, consider the case when, at a potential discontinuity, the characteristic on the left of the potential discontinuity is given as a constant velocity $\xi^{-}>0$. There are three possibilities (see Figure 2.1) :

1) $V^{-}>V^{+}$. In this case, the potential decreases, so the particle will cross the potential barrier and gain momentum in order to maintain a constant Hamiltonian. (2.4) implies

$$
\xi^{+}=\sqrt{\left(\xi^{-}\right)^{2}+2\left(V^{-}-V^{+}\right)} .
$$




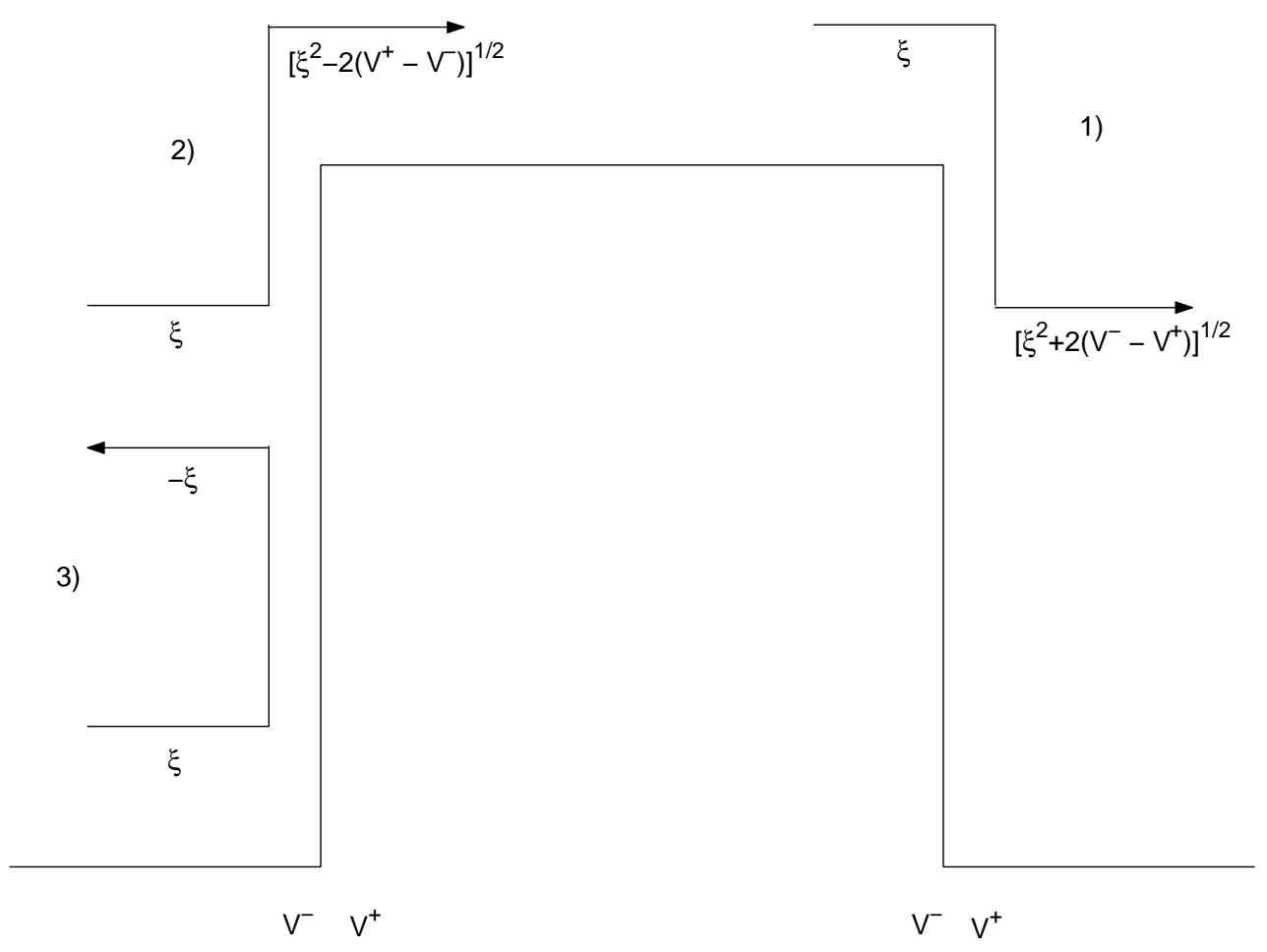

FIG. 2.1. Change of particle momentum across a potential barrier for the case when $\xi^{-}>0$.

2) $V^{-}<V^{+}$and $\frac{1}{2}\left(\xi^{-}\right)^{2}>V^{+}-V^{-}$. If the kinetic energy of the particle is bigger than the potential jump then the particle will cross the barrier with a reduced momentum. (2.4) implies

$$
\xi^{+}=\sqrt{\left(\xi^{-}\right)^{2}-2\left(V^{+}-V^{-}\right)} .
$$

3) $V^{-}<V^{+}$and $\frac{1}{2}\left(\xi^{-}\right)^{2}<V^{+}-V^{-}$. In this case, the kinetic energy is not large enough for the particle to cross the potential barrier, so the particle will be reflected with a negative velocity $-\xi^{-}$.

If $\xi^{-}<0$, similar behavior can also be analyzed using the constant Hamiltonian condition (2.4). See Fig.2.1.

The main ingredient in the well-balanced kinetic scheme by Perthame and Simeoni [21] for the shallow water equations with topography was to build in the above mechanism into the numerical scheme in order to preserve the steady state solution of the shallow water equations when the water velocity is zero. Note that the density distribution $f$ remains unchanged across the potential barrier, thus

$$
f\left(t, x^{+}, \xi^{+}\right)=f\left(t, x^{-}, \xi^{-}\right)
$$

at a discontinuous point $x$ of $V(x)$, where $\xi^{+}$and $\xi^{-}$are related by the constant Hamiltonian condition (2.4). This was used in constructing the numerical flux in [21].

In this paper, we use this mechanism for the numerical approximation to the Liouville equation with a discontinuous potential. This approximation, by its design, 
maintains a constant Hamiltonian up to approximation error across the potential barrier. The new issue faced here, not explored in [21], is the discretization in the $\xi$ direction. Given $\xi^{-}$as a grid point, the $\xi^{+}$constructed from the constant Hamiltonian condition (2.4) may not be a grid point, thus some appropriate interpolations in the $\xi$-direction are needed here. The approximation in the $\xi$-direction, and its consequent numerical properties, constitutes the main body of this paper.

\section{Scheme I: a finite difference approach}

3.1. The Hamiltonian-preserving numerical flux. We now describe our first finite difference scheme for the Liouville equation with a discontinuous potential. We call this scheme Scheme $I$.

Assume that the discontinuous points of potential $V$ are located at the grid points. Let the left and right limits of $V$ at point $x_{i+1 / 2}$ be $V_{i+\frac{1}{2}}^{+}$and $V_{i+\frac{1}{2}}^{-}$respectively. Note that if $V$ is continuous at $x_{j+1 / 2}$, then $V_{i+\frac{1}{2}}^{+}=V_{i+\frac{1}{2}}^{-}$. We approximate $V$ by a piecewise linear function

$$
V(x) \approx V_{i-1 / 2}^{+}+\frac{V_{i+1 / 2}^{-}-V_{i-1 / 2}^{+}}{\Delta x}\left(x-x_{i-1 / 2}\right) .
$$

We will adopt the flux splitting technique used in [21]. The semidiscrete scheme (with time continuous) reads

$$
\partial_{t} f_{i j}+\xi_{j} \frac{f_{i+\frac{1}{2}, j}^{-}-f_{i-\frac{1}{2}, j}^{+}}{\Delta x}-\frac{V_{i+\frac{1}{2}}^{-}-V_{i-\frac{1}{2}}^{+}}{\Delta x} \frac{f_{i, j+\frac{1}{2}}-f_{i, j-\frac{1}{2}}}{\Delta \xi}=0,
$$

where the numerical fluxes $f_{i, j+\frac{1}{2}}$ are defined using the upwind discretization. Since the characteristics of the Liouville equation may be different on the two sides of a potential discontinuity, the corresponding numerical fluxes should also be different. The essential part of our algorithm is to define the split numerical fluxes $f_{i+\frac{1}{2}, j}^{-}, f_{i-\frac{1}{2}, j}^{+}$ at each cell interface. We will use (2.5) to define these fluxes.

Assume $V$ is discontinuous at $x_{i+1 / 2}$. Consider the case $\xi_{j}>0$. Using the upwind scheme, $f_{i+\frac{1}{2}, j}^{-}=f_{i j}$. However,

$$
f_{i+1 / 2, j}^{+}=f\left(x_{i+1 / 2}^{+}, \xi_{j}^{+}\right)=f\left(x_{i+1 / 2}^{-}, \xi_{j}^{-}\right)
$$

while $\xi^{-}$is obtained from $\xi_{j}^{+}=\xi_{j}$ from (2.4). Since $\xi^{-}$may not be a grid point, we have to define it approximately. The first approach is to locate the two cell centers that bound this velocity, then use a linear interpolation to evaluate the needed numerical flux at $\xi^{-}$. The case of $\xi_{j}<0$ is treated similarly. The detailed algorithm to generate the numerical flux is given below.

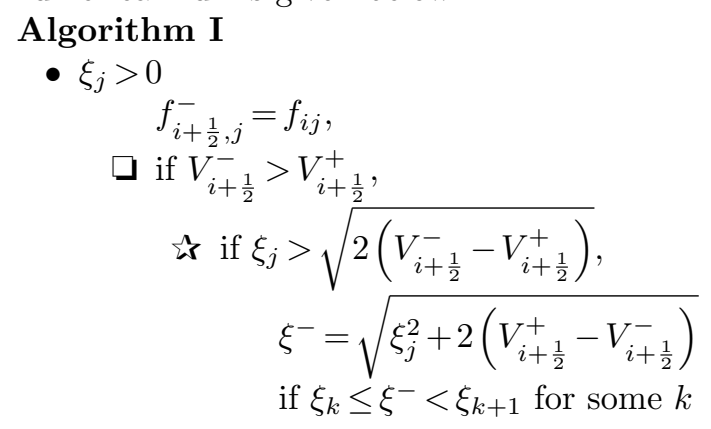


it else

$$
\text { then } f_{i+\frac{1}{2}, j}^{+}=\frac{\xi_{k+1}-\xi^{-}}{\Delta \xi} f_{i k}+\frac{\xi^{-}-\xi_{k}}{\Delta \xi} f_{i, k+1}
$$

is end

$$
f_{i+\frac{1}{2}, j}^{+}=f_{i+1, k} \text { where } \xi_{k}=-\xi_{j}
$$

$\square$ if $V_{i+\frac{1}{2}}^{-}<V_{i+\frac{1}{2}}^{+}$

$$
\begin{aligned}
& \xi^{-}=\sqrt{\xi_{j}^{2}+2\left(V_{i+\frac{1}{2}}^{+}-V_{i+\frac{1}{2}}^{-}\right)} \\
& \text {if } \xi_{k} \leq \xi^{-}<\xi_{k+1} \text { for some } k \\
& \text { then } f_{i+\frac{1}{2}, j}^{+}=\frac{\xi_{k+1}-\xi^{-}}{\Delta \xi} f_{i k}+\frac{\xi^{-}-\xi_{k}}{\Delta \xi} f_{i, k+1}
\end{aligned}
$$

$\square$ if $V_{i+\frac{1}{2}}^{-}=V_{i+\frac{1}{2}}^{+}$

$\square$ end

$$
f_{i+\frac{1}{2}, j}^{+}=f_{i+\frac{1}{2}, j}^{-}
$$

- $\xi_{j}<0$

$$
\begin{gathered}
f_{i+\frac{1}{2}, j}^{+}=f_{i+1, j}, \\
\square \text { if } V_{i+\frac{1}{2}}^{-}<V_{i+\frac{1}{2}}^{+}, \\
\text {it if }\left|\xi_{j}\right|>\sqrt{2\left(V_{i+\frac{1}{2}}^{+}-V_{i+\frac{1}{2}}^{-}\right)}, \\
\xi^{+}=-\sqrt{\xi_{j}^{2}+2\left(V_{i}-V_{i+\frac{1}{2}}^{+}\right)} \\
\text {if } \xi_{k} \leq \xi^{+}<\xi_{k+1} \text { for some } k \\
\text { then } f_{i+\frac{1}{2}, j}^{-}=\frac{\xi_{k+1}-\xi^{+}}{\Delta \xi} f_{i+1, k}+\frac{\xi^{+}-\xi_{k}}{\Delta \xi} f_{i+1, k+1} \\
\text { is else } \quad f_{i+\frac{1}{2}, j}^{-}=f_{i k} \text { where } \xi_{k}=-\xi_{j} \\
\text { if end } V_{i+\frac{1}{2}}^{-}>V_{i+\frac{1}{2}}^{+} \\
\xi^{+}=-\sqrt{\xi_{j}^{2}+2\left(V_{i+\frac{1}{2}}^{-}-V_{i+\frac{1}{2}}^{+}\right)} \\
\text {if } \xi_{k} \leq \xi^{+}<\xi_{k+1} \text { for some } k \\
\text { then } f_{i+\frac{1}{2}, j}^{-}=\frac{\xi_{k+1}-\xi^{+}}{\Delta \xi} f_{i+1, k}+\frac{\xi^{+}-\xi_{k}}{\Delta \xi} f_{i+1, k+1} \\
\square \text { if } V_{i+\frac{1}{2}}^{-}=V_{i+\frac{1}{2}}^{+} \\
f_{i+\frac{1}{2}, j}^{-}=f_{i+\frac{1}{2}, j}^{+} \\
\square \text { end }
\end{gathered}
$$

The above algorithm for evaluating numerical fluxes is of first order. One can obtain a second order flux by incorporating the slope limiter, such as van Leer or minmod slope limiter [17, 28], into the above algorithm. This can be achieved by replacing $f_{i k}$ by $f_{i k}+\frac{\Delta x}{2} s_{i k}$, and replacing $f_{i+1, k}$ by $f_{i+1, k}-\frac{\Delta x}{2} s_{i+1, k}$ in the above algorithm for all the possible index $k$, where $s_{i k}$ is the slope limiter in the $x$-direction.

After the spatial discretization is specified, one can use any time discretization for the time derivative.

3.2. Positivity and $l^{\infty}$-contraction. Since the exact solution of the Liouville equation is positive when the initial profile is, it is important that the numerical solution inherits this property.

We only consider the scheme using the first order numerical flux, and the for- 
ward Euler method in time. Without loss of generality, we consider the case $\xi_{j}>0$ and $V_{i+\frac{1}{2}}^{-}<V_{i-\frac{1}{2}}^{+}$for all $i$ (the other cases can be treated similarly with the same conclusion). The scheme reads

$$
\frac{f_{i j}^{n+1}-f_{i j}}{\Delta t}+\xi_{j} \frac{f_{i j}-\left(c_{1} f_{i-1, k}+c_{2} f_{i-1, k+1}\right)}{\Delta x}-\frac{V_{i+\frac{1}{2}}^{-}-V_{i-\frac{1}{2}}^{+}}{\Delta x} \frac{f_{i j}-f_{i, j-1}}{\Delta \xi}=0,
$$

where $c_{1}, c_{2}$ are positive and $c_{1}+c_{2}=1$. We omit the possible superscript $n$ of $f$. The above scheme can be rewritten as

$$
\begin{aligned}
f_{i j}^{n+1} & =\left(1-\left|\xi_{j}\right| \lambda_{x}^{t}-\frac{\left|V_{i+\frac{1}{2}}^{-}-V_{i-\frac{1}{2}}^{+}\right|}{\Delta x} \lambda_{\xi}^{t}\right) f_{i j}+\left|\xi_{j}\right| \lambda_{x}^{t}\left(c_{1} f_{i-1, k}+c_{2} f_{i-1, k+1}\right) \\
& +\frac{\left|V_{i+\frac{1}{2}}^{-}-V_{i-\frac{1}{2}}^{+}\right|}{\Delta x} \lambda_{\xi}^{t} f_{i, j-1} .
\end{aligned}
$$

Now we investigate the positivity of scheme (3.2). This is to prove that if $f_{i j}^{n} \geq 0$ for all $(i, j)$, then this is also true for $f^{n+1}$. Clearly one just needs to show that all the coefficients before $f^{n}$ are non-negative. A sufficient condition for this is clearly

$$
1-\left|\xi_{j}\right| \lambda_{x}^{t}-\frac{\left|V_{i+\frac{1}{2}}^{-}-V_{i-\frac{1}{2}}^{+}\right|}{\Delta x} \lambda_{\xi}^{t} \geq 0
$$

or

$$
\Delta t\left[\frac{\max _{j}\left|\xi_{j}\right|}{\Delta x}+\frac{\max _{i}\left|\frac{V_{i+\frac{1}{2}}^{-}-V_{i-\frac{1}{2}}^{+}}{\Delta x}\right|}{\Delta \xi}\right] \leq 1 .
$$

This CFL condition is similar to the CFL condition (2.3) of the usual finite difference scheme except that the quantity $\left|\frac{V_{i+\frac{1}{2}}^{-}-V_{i-\frac{1}{2}}^{+}}{\Delta x}\right|$ now represents the gradient of potential at its smooth point, which has a finite upper bound. Thus our new scheme has a hyperbolic CFL condition.

According to the study in [20], our second order scheme, which incorporates a slope limiter into the first order scheme, is positive under the half CFL condition, namely, the constant on the right hand side of (3.3) is $1 / 2$.

The above conclusion is analyzed based on the forward Euler time discretization. One can draw the same conclusion for the second order TVD Runge-Kutta time discretization [26].

The $l^{\infty}$-contracting property of this scheme

$$
\left\|f^{n+1}\right\|_{\infty} \leq\left\|f^{n}\right\|_{\infty} \quad \text { for any } \quad n
$$

follows easily with the same hyperbolic CFL condition, because the coefficients in (3.2) are positive and the sum of them is 1. 
3.3. The $l^{1}$-stability. The proof of $l^{1}$-stability is lengthy, and thus will be given in a separate paper [13]. Here we cite the main results of [13], which was established for the first order upwind flux with the forward Euler method in time, and a step function $V(x)$.

We first impose an assumption:

Assumption 1:

There exists a positive constant $\xi_{z}$ such that

$$
\forall(i, j) \in S_{z}=\left\{(i, j) \mid x_{i}<x_{m+\frac{1}{2}}, \quad 0<\xi_{j}<\xi_{z}\right\},
$$

it holds that

$$
\left|f_{i j}^{0}\right| \leq C_{1}\left|f^{0}\right|_{1} .
$$

We now state the following theorem from [13]:

Theorem 3.1. Under Assumption 1, there exists an $h_{0}>0$ such that, when $\Delta x<h_{0}$, Scheme I is $l^{1}$-stable:

$$
\left|f^{L}\right|_{1} \leq C\left|f^{0}\right|_{1} .
$$

REMARK 3.1. The semiclassical limit initial data (1.4) does not satisfy this condition. Thus Scheme I, when directly applied to this problem, may have stability problems, as shown in a counter example in [13]. However, if the decomposition idea mentioned in the Introduction is used, Scheme I is still suitable, which is what will be done in the numerical experiments of Section 8.

\section{Scheme II: a finite volume approach}

In this section another flux which results in an $l^{1}$-contracting scheme is proposed. We call this scheme Scheme II.

By integrating the Liouville equation (2.1) over the cell $\left[x_{i-1 / 2}, x_{i+1 / 2}\right] \times$ $\left[\xi_{j-1 / 2}, \xi_{j+1 / 2}\right]$, one gets the following equation

$$
\partial_{t} f_{i j}+\xi_{j} \frac{f_{i+\frac{1}{2}, j}^{-}-f_{i-\frac{1}{2}, j}^{+}}{\Delta x}-\frac{V_{i+\frac{1}{2}}^{-}-V_{i-\frac{1}{2}}^{+}}{\Delta x} \frac{f_{i, j+\frac{1}{2}}-f_{i, j-\frac{1}{2}}}{\Delta \xi}=0 .
$$

The upwind discretization depends on the sign of $\xi_{j}$ and $\frac{V_{i+\frac{1}{2}}^{-}-V_{i-\frac{1}{2}}^{+}}{\Delta x}$. To illustrate the basic idea, we assume $\xi_{j}>0, \frac{V_{i+\frac{1}{2}}^{-}-V_{i-\frac{1}{2}}^{+}}{\Delta x}<0$ and $V_{i+\frac{1}{2}}^{-}<V_{i+\frac{1}{2}}^{+}$(this is the case when the particle loses momentum from left to right at the barrier). In this case

$$
\begin{aligned}
& f_{i+\frac{1}{2}, j}^{-}=\frac{1}{\xi_{j} \Delta \xi} \int_{\xi_{j-\frac{1}{2}}}^{\xi_{j+\frac{1}{2}}} \xi f\left(x_{i+\frac{1}{2}}^{-}, \xi, t\right) d \xi \\
& f_{i, j+\frac{1}{2}}=\frac{1}{V_{i+\frac{1}{2}}^{-}-V_{i-\frac{1}{2}}^{+}} \int_{x_{i-\frac{1}{2}}}^{x_{i+\frac{1}{2}}} V_{x} f\left(x, \xi_{j+\frac{1}{2}}^{-}, t\right) d x .
\end{aligned}
$$

By using the condition (2.5):

$$
f_{i+\frac{1}{2}, j}^{+}=\frac{1}{\xi_{j} \Delta \xi} \int_{\xi_{j-\frac{1}{2}}}^{\xi_{j+\frac{1}{2}}} \xi f\left(x_{i+\frac{1}{2}}^{+}, \xi, t\right) d \xi=\frac{1}{\xi_{j} \Delta \xi} \int_{\xi_{j-\frac{1}{2}}}^{\xi_{j+\frac{1}{2}}} \xi \bar{f}\left(x_{i+\frac{1}{2}}^{-}, \xi, t\right) d \xi,
$$


where $\bar{f}$ is defined as

$$
\bar{f}\left(x_{i+\frac{1}{2}}^{-}, \xi, t\right)=f\left(x_{i+\frac{1}{2}}^{-}, \sqrt{\xi^{2}+2\left(V_{i+\frac{1}{2}}^{+}-V_{i+\frac{1}{2}}^{-}\right)}, t\right) .
$$

Using a change of variable on (4.2) leads to

$$
\begin{aligned}
f_{i+\frac{1}{2}, j}^{+} & =\frac{1}{\xi_{j} \Delta \xi} \int_{\xi_{j-\frac{1}{2}}}^{\xi_{j+\frac{1}{2}}} \xi f\left(x_{i+\frac{1}{2}}^{-}, \sqrt{\xi^{2}+2\left(V_{i+\frac{1}{2}}^{+}-V_{i+\frac{1}{2}}^{-}\right)}, t\right) d \xi \\
& =\frac{1}{\xi_{j} \Delta \xi} \int_{\xi_{1}^{\prime}}^{\xi_{2}^{2}} \xi f\left(x_{i+\frac{1}{2}}^{-}, \xi, t\right) d \xi,
\end{aligned}
$$

where

$$
\xi_{1}^{\prime}=\sqrt{\xi_{j-\frac{1}{2}}^{2}+2\left(V_{i+\frac{1}{2}}^{+}-V_{i+\frac{1}{2}}^{-}\right)}, \quad \xi_{2}^{\prime}=\sqrt{\xi_{j+\frac{1}{2}}^{2}+2\left(V_{i+\frac{1}{2}}^{+}-V_{i+\frac{1}{2}}^{-}\right)} .
$$

The integral in (4.3) will be approximated by a quadrature rule, since the end point $\xi_{1}^{\prime}$ and $\xi_{2}^{\prime}$ may not be grid points in the $\xi$-direction. We first need to locate the grid points that bound $\xi_{1}^{\prime}$ and $\xi_{2}^{\prime}$. There are two possibilities, namely, either $\xi_{1}^{\prime}$ and $\xi_{2}^{\prime}$ fall into the same cell, or they are in separate cells. In the former case, we use the midpoint rule. In the second case, the composite midpoint rule is used.

We propose the following evaluation of the split fluxes $f_{i+\frac{1}{2}, j}^{ \pm}$in (4.1).

\section{Algorithm II}

- if $\xi_{j}>0$

$$
f_{i+\frac{1}{2}, j}^{-}=f_{i j}
$$

$\square$ if $V_{i+\frac{1}{2}}^{-}>V_{i+\frac{1}{2}}^{+}$,

$$
\begin{aligned}
& \text { ts if } \xi_{j-\frac{1}{2}} \geq \sqrt{2\left(V_{i+\frac{1}{2}}^{-}-V_{i+\frac{1}{2}}^{+}\right)} \text {, } \\
& \xi_{1}^{\prime}=\sqrt{\xi_{j-\frac{1}{2}}^{2}-2\left(V_{i+\frac{1}{2}}^{-}-V_{i+\frac{1}{2}}^{+}\right)} \\
& \xi_{2}^{\prime}=\sqrt{\xi_{j+\frac{1}{2}}^{2}-2\left(V_{i+\frac{1}{2}}^{-}-V_{i+\frac{1}{2}}^{+}\right)} \\
& f_{i+\frac{1}{2}, j}^{+}=\frac{1}{\xi_{j}} \frac{\xi_{2}^{\prime}-\xi_{1}^{\prime}}{\Delta \xi} \xi_{k} f_{i k}
\end{aligned}
$$

else $\xi_{k-\frac{1}{2}} \leq \xi_{1}^{\prime}<\xi_{k+\frac{1}{2}}<\cdots<\xi_{k+s-\frac{1}{2}}<\xi_{2}^{\prime} \leq \xi_{k+s+\frac{1}{2}}$ for some $k, s$

$$
\begin{aligned}
& f_{i+\frac{1}{2}, j}^{+}=\frac{1}{\xi_{j}}\left\{\frac{\xi_{k+\frac{1}{2}}-\xi_{1}^{\prime}}{\Delta \xi} \xi_{k} f_{i k}+\xi_{k+1} f_{i, k+1}+\cdots\right. \\
& \left.+\xi_{k+s-1} f_{i, k+s-1}+\frac{\xi_{2}^{\prime}-\xi_{k+s-\frac{1}{2}}}{\Delta \xi} \xi_{k+s} f_{i, k+s}\right\}
\end{aligned}
$$

end

it else

$$
\text { it end }
$$

$$
f_{i+\frac{1}{2}, j}^{+}=f_{i+1, k} \text { where } \xi_{k}=-\xi_{j}
$$

$\square$ if $V_{i+\frac{1}{2}}^{-}<V_{i+\frac{1}{2}}^{+}$

$$
\xi_{1}^{\prime}=\sqrt{\xi_{j-\frac{1}{2}}^{2}+2\left(V_{i+\frac{1}{2}}^{+}-V_{i+\frac{1}{2}}^{-}\right)}
$$




$$
\xi_{2}^{\prime}=\sqrt{\xi_{j+\frac{1}{2}}^{2}+2\left(V_{i+\frac{1}{2}}^{+}-V_{i+\frac{1}{2}}^{-}\right)}
$$

if $\xi_{k-\frac{1}{2}} \leq \xi_{1}^{\prime}<\xi_{2}^{\prime} \leq \xi_{k+\frac{1}{2}}$ for some $k$

$$
f_{i+\frac{1}{2}, j}^{+}=\frac{1}{\xi_{j}} \frac{\xi_{2}^{\prime}-\xi_{1}^{\prime}}{\Delta \xi} \xi_{k} f_{i k}
$$

else $\xi_{k-\frac{1}{2}} \leq \xi_{1}^{\prime}<\xi_{k+\frac{1}{2}}<\cdots<\xi_{k+s-\frac{1}{2}}<\xi_{2}^{\prime} \leq \xi_{k+s+\frac{1}{2}}$ for some $k, s$

$$
\begin{aligned}
& f_{i+\frac{1}{2}, j}^{+}=\frac{1}{\xi_{j}}\left\{\frac{\xi_{k+\frac{1}{2}}-\xi_{1}^{\prime}}{\Delta \xi} \xi_{k} f_{i k}+\xi_{k+1} f_{i, k+1}+\cdots\right. \\
& \left.+\xi_{k+s-1} f_{i, k+s-1}+\frac{\xi_{2}^{\prime}-\xi_{k+s-\frac{1}{2}}}{\Delta \xi} \xi_{k+s} f_{i, k+s}\right\}
\end{aligned}
$$

end

$$
\begin{gathered}
\square \text { if } V_{i+\frac{1}{2}}^{-}=V_{i+\frac{1}{2}}^{+} \\
\quad f_{i+\frac{1}{2}, j}^{+}=f_{i+\frac{1}{2}, j}^{-} \\
\square \text { end }
\end{gathered}
$$

- if $\xi_{j}<0$

$$
f_{i+\frac{1}{2}, j}^{+}=f_{i+1, j}
$$

$\square$ if $V_{i+\frac{1}{2}}^{-}<V_{i+\frac{1}{2}}^{+}$

$$
\begin{aligned}
& \text { if }\left|\xi_{j+\frac{1}{2}}\right|>\sqrt{2\left(V_{i+\frac{1}{2}}^{+}-V_{i+\frac{1}{2}}^{-}\right)}, \\
& \xi_{1}^{\prime}=-\sqrt{\xi_{j-\frac{1}{2}}^{2}+2\left(V_{i+\frac{1}{2}}^{-}-V_{i+\frac{1}{2}}^{+}\right)} \\
& \xi_{2}^{\prime}=-\sqrt{\xi_{j+\frac{1}{2}}^{2}+2\left(V_{i+\frac{1}{2}}^{-}-V_{i+\frac{1}{2}}^{+}\right)} \\
& \text {if } \xi_{k-\frac{1}{2}} \leq \xi_{1}^{\prime}<\xi_{2}^{\prime} \leq \xi_{k+\frac{1}{2}} \text { for some } k \\
& f_{i+\frac{1}{2}, j}^{-}=\frac{1}{\xi_{j}} \frac{\xi_{2}^{\prime}-\xi_{1}^{\prime}}{\Delta \xi} \xi_{k} f_{i+1, k}
\end{aligned}
$$

粰 $\xi_{k-\frac{1}{2}} \leq \xi_{1}^{\prime}<\xi_{k+\frac{1}{2}}<\cdots<\xi_{k+s-\frac{1}{2}}<\xi_{2}^{\prime} \leq \xi_{k+s+\frac{1}{2}}$ for some $k, s$

$$
\begin{aligned}
& f_{i+\frac{1}{2}, j}^{-}=\frac{1}{\xi_{j}}\left\{\frac{\xi_{k+\frac{1}{2}}-\xi_{1}^{\prime}}{\Delta \xi} \xi_{k} f_{i+1, k}+\xi_{k+1} f_{i+1, k+1}+\cdots\right. \\
& \left.+\xi_{k+s-1} f_{i+1, k+s-1}+\frac{\xi_{2}^{\prime}-\xi_{k+s-\frac{1}{2}}}{\Delta \xi} \xi_{k+s} f_{i+1, k+s}\right\}
\end{aligned}
$$

end

is else

is end

$$
f_{i+\frac{1}{2}, j}^{-}=f_{i k} \text { where } \xi_{k}=-\xi_{j}
$$

$\square$ if $V_{i+\frac{1}{2}}^{-}>V_{i+\frac{1}{2}}^{+}$

$$
\begin{gathered}
\xi_{1}^{\prime}=-\sqrt{\xi_{j-\frac{1}{2}}^{2}+2\left(V_{i+\frac{1}{2}}^{-}-V_{i+\frac{1}{2}}^{+}\right)} \\
\xi_{2}^{\prime}=-\sqrt{\xi_{j+\frac{1}{2}}^{2}+2\left(V_{i+\frac{1}{2}}^{-}-V_{i+\frac{1}{2}}^{+}\right)} \\
\text {if } \xi_{k-\frac{1}{2}} \leq \xi_{1}^{\prime}<\xi_{2}^{\prime} \leq \xi_{k+\frac{1}{2}} \text { for some } k \\
f_{i+\frac{1}{2}, j}^{-}=\frac{1}{\xi_{j}} \frac{\xi_{2}^{\prime}-\xi_{1}^{\prime}}{\Delta \xi} \xi_{k} f_{i+1, k}
\end{gathered}
$$

else $\xi_{k-\frac{1}{2}} \leq \xi_{1}^{\prime}<\xi_{k+\frac{1}{2}}<\cdots<\xi_{k+s-\frac{1}{2}}<\xi_{2}^{\prime} \leq \xi_{k+s+\frac{1}{2}}$ for some $k, s$

$$
\begin{aligned}
& f_{i+\frac{1}{2}, j}^{-}=\frac{1}{\xi_{j}}\left\{\frac{\xi_{k+\frac{1}{2}}-\xi_{1}^{\prime}}{\Delta \xi} \xi_{k} f_{i+1, k}+\xi_{k+1} f_{i+1, k+1}+\cdots\right. \\
& \left.+\xi_{k+s-1} f_{i+1, k+s-1}+\frac{\xi_{2}^{\prime}-\xi_{k+s-\frac{1}{2}}}{\Delta \xi} \xi_{k+s} f_{i+1, k+s}\right\}
\end{aligned}
$$


橉 end

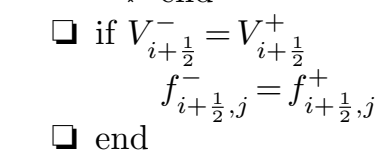

- end

REMARK 4.1. The above Algorithm uses a first order quadrature rule at the ends of the interval (4.3), thus it is of first order even if the slope limiters in $x$-direction are incorporated into the algorithm. One can also use a second order quadrature rule at the ends of intervals (4.3).

\section{Stability theory of Scheme II}

In this section we study the $l^{1}$ and $l^{\infty}$ stability of Scheme II. Its positivity is obvious under the hyperbolic CFL condition (3.3).

THEOREM 5.1. If the forward Euler time discretization is used, then the flux given by Algorithm II yields the scheme (4.1) which is $l^{1}$-contracting and $l^{\infty}$-stable.

Proof. For simplicity, we discuss the case when the potential has only one discontinuity at grid point $x_{m+\frac{1}{2}}$ with jump $V_{m+\frac{1}{2}}^{-}-V_{m+\frac{1}{2}}^{+}=D>0$, and $V^{\prime}(x)<0$ at smooth points. The other cases, namely, when $V^{\prime}(x) \geq 0$, or the potential having several discontinuity points with increased or decreased potential jumps, can be discussed similarly.

We consider the typical situation when $\xi_{1}<-\sqrt{2 D}, \xi_{M}>\sqrt{2 D}$, so that all the three situations discussed in Section 2 are included. We assume the mesh is defined such that $0, \pm \sqrt{2 D}$ are grid points in the $\xi$-direction. We define some sets of indexes

$$
\begin{aligned}
& D_{m}^{+}=\left\{(m, j) \mid \xi_{j}>0\right\}, \\
& D_{m+1}^{+}=\left\{(m+1, j) \mid \xi_{j-\frac{1}{2}} \geq \sqrt{2 D}\right\}, \\
& D_{m}^{-}=\left\{(m, j) \mid-\sqrt{\xi_{1}^{2}-2 D} \leq \xi_{j-\frac{1}{2}} \leq 0\right\}, \\
& D_{m+1}^{-}=\left\{(m+1, j) \mid \xi_{j+\frac{1}{2}} \leq-\sqrt{2 D}\right\}, \\
& D_{l}^{4}=\left\{(i, j) \mid x_{i} \leq x_{m}, \xi_{j+\frac{1}{2}} \leq-\sqrt{\xi_{1}^{2}-2 D}\right\} .
\end{aligned}
$$

These domains are shown in Figure 5.1.

Due to velocity change across the potential jump at $x_{m+\frac{1}{2}}, D_{l}^{4}$ represents the area where particles come from outside of the domain $\left[x_{1}, x_{N}\right] \times\left[\xi_{1}, \xi_{M}\right]$. In order to implement Scheme I conveniently, we need to choose the computational domain as

$$
E_{d}=\{(i, j) \mid i=1, \cdots, N, j=1, \cdots, M\} \backslash D_{l}^{4} .
$$

Now denote $F_{i}=\frac{1}{\Delta x}\left|V_{i+\frac{1}{2}}^{-}-V_{i-\frac{1}{2}}^{+}\right|$. Our scheme (4.1) with Algorithm II can be made precisely as

1) if $\xi_{j}>0, i \neq m+1$,

$$
f_{i j}^{n+1}=\left(1-F_{i} \lambda_{\xi}^{t}-\xi_{j} \lambda_{x}^{t}\right) f_{i j}+F_{i} \lambda_{\xi}^{t} f_{i, j-1}+\xi_{j} \lambda_{x}^{t} f_{i-1, j} ;
$$

2) if $\xi_{j}<0, i \neq m$,

$$
f_{i j}^{n+1}=\left(1-F_{i} \lambda_{\xi}^{t}-\left|\xi_{j}\right| \lambda_{x}^{t}\right) f_{i j}+F_{i} \lambda_{\xi}^{t} f_{i, j-1}+\left|\xi_{j}\right| \lambda_{x}^{t} f_{i+1, j} ;
$$




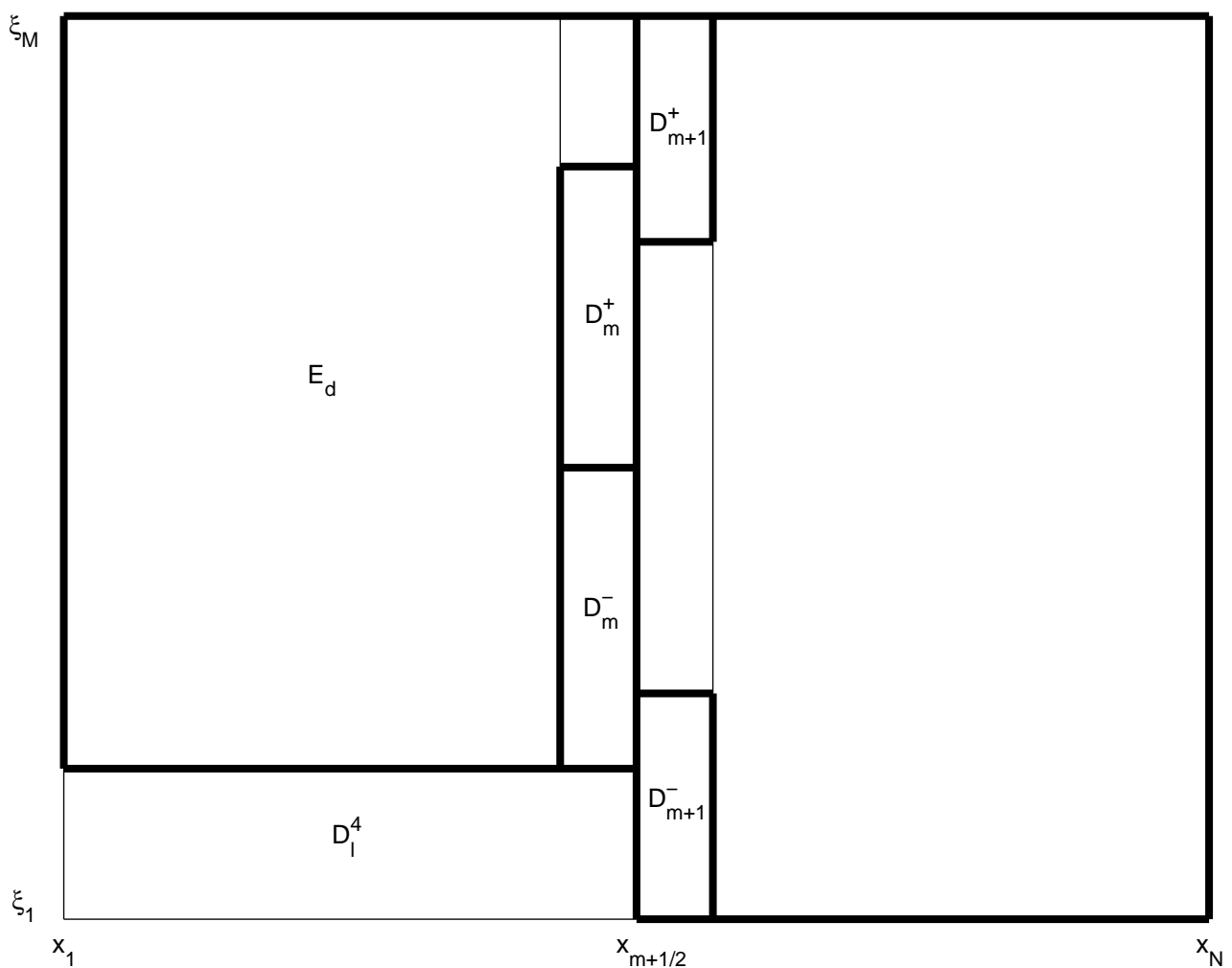

FIG. 5.1. Sketch of the index sets $D_{m}^{+}, D_{m+1}^{+}, D_{m}^{-}, D_{m+1}^{-}, D_{l}^{4}$.

3) if $\xi_{j}>0$,

$$
f_{m+1, j}^{n+1}=\left(1-F_{m+1} \lambda_{\xi}^{t}-\xi_{j} \lambda_{x}^{t}\right) f_{m+1, j}+F_{m+1} \lambda_{\xi}^{t} f_{m+1, j-1}+\xi_{j} \lambda_{x}^{t} f_{m+\frac{1}{2}, j}^{+}
$$

4) if $\xi_{j}<0$,

$$
f_{m j}^{n+1}=\left(1-F_{m} \lambda_{\xi}^{t}-\left|\xi_{j}\right| \lambda_{x}^{t}\right) f_{m j}+F_{m} \lambda_{\xi}^{t} f_{m, j-1}+\left|\xi_{j}\right| \lambda_{x}^{t} f_{m+\frac{1}{2}, j}^{-},
$$

where we omit the superscript $n$ on the right hand side.

Due to the linearity of the scheme, the equation for the error between the analytical and the numerical solution is the same as the scheme itself, so in this section, $f_{i j}$ will denote the error. We assume there is no error at the boundary, thus $f_{i j}^{n}=0$ at the boundary. If the $l^{1}$-norm of the error introduced at each time step in incoming boundary cells is ensured to be $o(1)$ part of $\left|f^{n}\right|_{1}$, our following analysis still applies.

By summing up (5.1)-(5.4) for $(i, j) \in E_{d}$, one typically gets the following expression

$$
\begin{aligned}
\sum_{(i, j) \in E_{d}} f_{i j}^{n+1} & =\sum_{(i, j) \in E_{d}} \alpha_{i j} f_{i j}+\sum_{(m+1, j) \in D_{m+1}^{+}} \xi_{j} \lambda_{x}^{t} f_{m+\frac{1}{2}, j}^{+}+\sum_{(m, j) \in D_{m}^{-}} \xi_{j} \lambda_{x}^{t} f_{m+\frac{1}{2}, j}^{-} \\
& \equiv I_{1}+I_{2}+I_{3}
\end{aligned}
$$


Due to the zero boundary condition, the coefficients $\alpha_{i j}$ in (5.5) satisfy

$$
\begin{aligned}
& \alpha_{i j} \leq 1, \quad(i, j) \in E_{d} \backslash\left\{D_{m}^{+} \cup D_{m+1}^{-}\right\}, \\
& \alpha_{i j} \leq 1-\left|\xi_{j}\right| \lambda_{x}^{t}, \quad(i, j) \in D_{m}^{+} \cup D_{m+1}^{-} .
\end{aligned}
$$

We now study the relation between $I_{2}$ and $\sum_{(m, j) \in D_{m}^{+}}\left|\xi_{j} \lambda_{x}^{t} f_{m j}\right|$. Let

$$
p_{N+1}=\sqrt{\xi_{N+\frac{1}{2}}^{2}-2 D},
$$

and assume

$$
\xi_{k}<p_{N+1} \leq \xi_{k+1} \leq \xi_{N+\frac{1}{2}} .
$$

Assume $\xi_{J_{2}-\frac{1}{2}}=0$ for some $J_{2}$. Since

$$
\frac{1}{\lambda_{x}^{t}} I_{2} \leq \sum_{j=J_{2}}^{k-1}\left|\xi_{j} f_{m j}\right|+\frac{p_{N+1}-\xi_{k}}{\Delta \xi}\left|\xi_{k} f_{m k}\right| \leq \sum_{(m, j) \in D_{m}^{+}}\left|\xi_{j} f_{m j}\right|,
$$

thus

$$
I_{2} \leq \sum_{(m, j) \in D_{m}^{+}}\left|\xi_{j} \lambda_{x}^{t} f_{m j}\right|
$$

Similarly, one gets

$$
I_{3} \leq \sum_{(m+1, j) \in D_{m+1}^{-}}\left|\xi_{j} \lambda_{x}^{t} f_{m+1, j}\right| .
$$

Combining (5.5), (5.6), (5.7), (5.8) and (5.9) gives

$$
\sum_{(i, j) \in E_{d}}\left|f_{i j}^{n+1}\right| \leq \sum_{(i, j) \in E_{d}}\left|f_{i j}^{n}\right| .
$$

This is the $l^{1}$-contracting property of this scheme.

Next we prove the $l^{\infty}$-stability. In the cells where $\xi_{j}>0, i \neq m+1$ or $\xi_{j}<0, i \neq m$, one uses (5.1) or (5.2). Observing that the coefficients on the right hand side of (5.1) or (5.2) are positive and the sum of them is 1 , so in fact these schemes are $l^{\infty}$-contracting. It remains to study the cells where $\xi_{j}>0, i=m+1$ or $\xi_{j}<0, i=m$, corresponding to $(5.3)$ or $(5.4)$.

We first consider (5.3). It can be checked that when $\xi_{j-\frac{1}{2}}<\sqrt{2 D}, f_{m+\frac{1}{2}, j}^{+}=f_{m+1, k}$ with $k$ such that $\xi_{k}=-\xi_{j}$, thus the $l^{\infty}$-contracting property still holds. When $\xi_{j-\frac{1}{2}} \geq$ $\sqrt{2 D}$, denote

$$
\xi_{1}^{\prime}=\sqrt{\left(\xi_{j-\frac{1}{2}}\right)^{2}-2 D}, \quad \xi_{2}^{\prime}=\sqrt{\left(\xi_{j+\frac{1}{2}}\right)^{2}-2 D}
$$

Since $V_{m+\frac{1}{2}}^{+}<V_{m+\frac{1}{2}}^{-}$, one has $\xi_{2}^{\prime}-\xi_{1}^{\prime}>\Delta \xi$. Therefore, it is impossible that $\xi_{k-\frac{1}{2}} \leq$ $\xi_{1}^{\prime}<\xi_{2}^{\prime} \leq \xi_{k+\frac{1}{2}}$ for any $k$. Assume $\xi_{k-\frac{1}{2}} \leq \xi_{1}^{\prime}<\xi_{k+\frac{1}{2}}<\cdots<\xi_{k+s-\frac{1}{2}}<\xi_{2}^{\prime} \leq \xi_{k+s+\frac{1}{2}}$ with 
PHI IN AND KIN WEN

299

$s \geq 1$. In this case

$$
\begin{aligned}
f_{m+\frac{1}{2}, j}^{+}= & \frac{1}{\xi_{j}}\left\{\frac{\xi_{k+\frac{1}{2}}-\xi_{1}^{\prime}}{\Delta \xi} \xi_{k} f_{m k}+\xi_{k+1} f_{m, k+1}+\cdots\right. \\
& \left.+\xi_{k+s-1} f_{m, k+s-1}+\frac{\xi_{2}^{\prime}-\xi_{k+s-\frac{1}{2}}}{\Delta \xi} \xi_{k+s} f_{m, k+s}\right\} .
\end{aligned}
$$

Substituting (5.12) into (5.3) yields

$$
\begin{aligned}
f_{m+1, j}^{n+1}= & \left(1-F_{m+1} \lambda_{\xi}^{t}-\xi_{j} \lambda_{x}^{t}\right) f_{m+1, j}+F_{m+1} \lambda_{\xi}^{t} f_{m+1, j-1} \\
+ & \lambda_{x}^{t}\left\{\frac{\xi_{k+\frac{1}{2}}-\xi_{1}^{\prime}}{\Delta \xi} \xi_{k} f_{m k}+\xi_{k+1} f_{m, k+1}+\cdots\right. \\
& \left.+\xi_{k+s-1} f_{m, k+s-1}+\frac{\xi_{2}^{\prime}-\xi_{k+s-\frac{1}{2}}}{\Delta \xi} \xi_{k+s} f_{m, k+s}\right\} .
\end{aligned}
$$

Observing that the coefficients on the right hand side of (5.13) are still positive. Thus it remains to check the sum of these coefficients given by

$$
\begin{aligned}
I_{4} & =1-\xi_{j} \lambda_{x}^{t}+\lambda_{x}^{t}\left\{\frac{\xi_{k+\frac{1}{2}}-\xi_{1}^{\prime}}{\Delta \xi} \xi_{k}+\xi_{k+1}+\cdots+\xi_{k+s-1}+\frac{\xi_{2}^{\prime}-\xi_{k+s-\frac{1}{2}}}{\Delta \xi} \xi_{k+s}\right\} \\
& =1+\xi_{j} \lambda_{x}^{t}\left\{\frac{\frac{\xi_{k+\frac{1}{2}}-\xi_{1}^{\prime}}{\Delta \xi} \xi_{k}+\xi_{k+1}+\cdots+\xi_{k+s-1}+\frac{\xi_{2}^{\prime}-\xi_{k+s-\frac{1}{2}}}{\Delta \xi} \xi_{k+s}}{\xi_{j}}-1\right\} .
\end{aligned}
$$

Let

$$
\begin{aligned}
& \xi_{k}^{\prime}=\frac{\xi_{k}+\xi_{k+s}}{2}=\frac{\xi_{k+\frac{1}{2}}+\xi_{k+s-\frac{1}{2}}}{2}, \\
& a_{1}^{\prime}=\frac{\xi_{k+\frac{1}{2}}-\xi_{1}^{\prime}}{\Delta \xi} \in(0,1], \\
& a_{2}^{\prime}=\frac{\xi_{2}^{\prime}-\xi_{k+s-\frac{1}{2}}}{\Delta \xi} \in(0,1],
\end{aligned}
$$

then

$$
\xi_{k}=\xi_{k}^{\prime}-\frac{s \Delta \xi}{2}, \quad \xi_{k+s}=\xi_{k}^{\prime}+\frac{s \Delta \xi}{2}
$$

Also notice

$$
\xi_{k}^{\prime}-\frac{1}{2}\left(\xi_{1}^{\prime}+\xi_{2}^{\prime}\right)=\frac{1}{2}\left[\left(\xi_{k+\frac{1}{2}}-\xi_{1}^{\prime}\right)+\left(\xi_{k+s-\frac{1}{2}}-\xi_{2}^{\prime}\right)\right]=\frac{1}{2}\left(a_{1}^{\prime}-a_{2}^{\prime}\right) \Delta \xi
$$


hence one gets

$$
\begin{aligned}
& \frac{1}{\xi_{j}}\left\{\frac{\xi_{k+\frac{1}{2}}-\xi_{1}^{\prime}}{\Delta \xi} \xi_{k}+\xi_{k+1}+\cdots+\xi_{k+s-1}+\frac{\xi_{2}^{\prime}-\xi_{k+s-\frac{1}{2}}}{\Delta \xi} \xi_{k+s}\right\} \\
= & \frac{1}{\xi_{j}}\left\{\frac{\xi_{k+\frac{1}{2}}-\xi_{1}^{\prime}}{\Delta \xi} \xi_{k}^{\prime}+\xi_{k}^{\prime}+\cdots+\xi_{k}^{\prime}+\frac{\xi_{2}^{\prime}-\xi_{k+s-\frac{1}{2}}}{\Delta \xi} \xi_{k}^{\prime}\right\}+\frac{s \Delta \xi}{2 \xi_{j}}\left(a_{2}^{\prime}-a_{1}^{\prime}\right) \\
= & \frac{\left(\xi_{2}^{\prime}-\xi_{1}^{\prime}\right) \xi_{k}^{\prime}}{\xi_{j} \Delta \xi}+\frac{s \Delta \xi}{2 \xi_{j}}\left(a_{2}^{\prime}-a_{1}^{\prime}\right) \\
= & \frac{\left(\xi_{2}^{\prime}-\xi_{1}^{\prime}\right)\left(\xi_{1}^{\prime}+\xi_{2}^{\prime}\right)}{2 \xi_{j} \Delta \xi}+\frac{s \Delta \xi}{2 \xi_{j}}\left(a_{2}^{\prime}-a_{1}^{\prime}\right)-\frac{\left(\xi_{2}^{\prime}-\xi_{1}^{\prime}\right)}{2 \xi_{j}}\left(a_{2}^{\prime}-a_{1}^{\prime}\right) \\
= & \frac{\left(\xi_{2}^{\prime 2}-\xi_{1}^{\prime 2}\right)}{\left(\xi_{j+\frac{1}{2}}-\xi_{j-\frac{1}{2}}\right)\left(\xi_{j+\frac{1}{2}}+\xi_{j-\frac{1}{2}}\right)}+\frac{\left(1-a_{1}^{\prime}-a_{2}^{\prime}\right) \Delta \xi}{2 \xi_{j}}\left(a_{2}^{\prime}-a_{1}^{\prime}\right) \\
< & 1+\frac{\Delta \xi}{8 \xi_{j}}<1+\frac{\Delta \xi}{8 \sqrt{2 D}} .
\end{aligned}
$$

Substituting (5.15) into (5.14), the sum of the coefficients in (5.13) is estimated as

$$
I_{4}<1+\xi_{j} \lambda_{x}^{t} \frac{\Delta \xi}{8 \sqrt{2 D}}<1+\frac{1}{8 \lambda_{\xi}^{t} \sqrt{2 d}} \Delta t
$$

where the last inequality is deduced by using the CFL condition.

Now we consider case (5.4). Denote

$$
\xi_{1}^{\prime}=-\sqrt{\left(\xi_{j-\frac{1}{2}}\right)^{2}+2 D}, \quad \xi_{2}^{\prime}=-\sqrt{\left(\xi_{j+\frac{1}{2}}\right)^{2}+2 D} .
$$

In this case, $\xi_{2}^{\prime}-\xi_{1}^{\prime}<\Delta \xi$. So there are two cases $\xi_{k-\frac{1}{2}} \leq \xi_{1}^{\prime}<\xi_{2}^{\prime} \leq \xi_{k+\frac{1}{2}}$ or $\xi_{k-\frac{1}{2}} \leq \xi_{1}^{\prime}<$ $\xi_{k+\frac{1}{2}}<\xi_{2}^{\prime} \leq \xi_{k+\frac{3}{2}}$ corresponding respectively to

$$
f_{m+\frac{1}{2}, j}^{-}=\frac{1}{\xi_{j}} \frac{\xi_{2}^{\prime}-\xi_{1}^{\prime}}{\Delta \xi} \xi_{k} f_{m+1, k}
$$

or

$$
f_{m+\frac{1}{2}, j}^{-}=\frac{1}{\xi_{j}}\left\{\frac{\xi_{k+\frac{1}{2}}-\xi_{1}^{\prime}}{\Delta \xi} \xi_{k} f_{m+1, k}+\frac{\xi_{2}^{\prime}-\xi_{k+\frac{1}{2}}}{\Delta \xi} \xi_{k+1} f_{m+1, k+1}\right\} .
$$

Substituting (5.18) or (5.19) into (5.4) gives

$$
f_{m j}^{n+1}=\left(1-F_{m} \lambda_{\xi}^{t}-\left|\xi_{j}\right| \lambda_{x}^{t}\right) f_{m j}+F_{m} \lambda_{\xi}^{t} f_{m, j-1}+\lambda_{x}^{t} \frac{\xi_{2}^{\prime}-\xi_{1}^{\prime}}{\Delta \xi}\left|\xi_{k}\right| f_{m+1, k}
$$

or

$$
\begin{aligned}
f_{m j}^{n+1} & =\left(1-F_{m} \lambda_{\xi}^{t}-\left|\xi_{j}\right| \lambda_{x}^{t}\right) f_{m j}+F_{m} \lambda_{\xi}^{t} f_{m, j-1} \\
& +\lambda_{x}^{t}\left\{\frac{\xi_{k+\frac{1}{2}}-\xi_{1}^{\prime}}{\Delta \xi}\left|\xi_{k}\right| f_{m+1, k}+\frac{\xi_{2}^{\prime}-\xi_{k+\frac{1}{2}}}{\Delta \xi}\left|\xi_{k+1}\right| f_{m+1, k+1}\right\} .
\end{aligned}
$$


In each case, the coefficients on the right hand side are positive. Thus it remains to check the sum of the coefficients, which is, respectively,

$$
1+\left|\xi_{j}\right| \lambda_{x}^{t}\left\{\frac{\left(\xi_{2}^{\prime}-\xi_{1}^{\prime}\right)\left|\xi_{k}\right|}{\Delta \xi\left|\xi_{j}\right|}-1\right\}
$$

or

$$
1+\left|\xi_{j}\right| \lambda_{x}^{t}\left\{\frac{\left(\xi_{k+\frac{1}{2}}-\xi_{1}^{\prime}\right)\left|\xi_{k}\right|+\left(\xi_{2}^{\prime}-\xi_{k+\frac{1}{2}}\right)\left|\xi_{k+1}\right|}{\Delta \xi\left|\xi_{j}\right|}-1\right\} .
$$

Let $D_{k}$ be $\frac{\left(\xi_{2}^{\prime}-\xi_{1}^{\prime}\right)\left|\xi_{k}\right|}{\Delta \xi\left|\xi_{j}\right|}$ or $\frac{\left(\xi_{k+\frac{1}{2}}-\xi_{1}^{\prime}\right)\left|\xi_{k}\right|+\left(\xi_{2}^{\prime}-\xi_{k+\frac{1}{2}}\right)\left|\xi_{k+1}\right|}{\Delta \xi\left|\xi_{j}\right|}$. One has

$$
D_{k}<\frac{\left(\xi_{2}^{\prime}-\xi_{1}^{\prime}\right)\left[\frac{1}{2}\left(\left|\xi_{1}^{\prime}\right|+\left|\xi_{2}^{\prime}\right|\right)+\Delta \xi\right]}{\Delta \xi\left|\xi_{j}\right|}=\frac{\frac{1}{2}\left(\left|\xi_{1}^{\prime}\right|+\left|\xi_{2}^{\prime}\right|\right)+\Delta \xi}{\frac{1}{2}\left(\left|\xi_{1}^{\prime}\right|+\left|\xi_{2}^{\prime}\right|\right)}<1+\frac{\Delta t}{\lambda_{\xi}^{t} \sqrt{2 D}}
$$

thus the sums (5.22) and (5.23) are both bounded above by

$$
1+\frac{1}{\lambda_{\xi}^{t} \sqrt{2 D}} \Delta t
$$

Combining (5.16) and (5.24), and letting $C_{0}=\frac{1}{\lambda_{\xi}^{t} \sqrt{2 D}}$ be an $O(1)$ quantity, we get

$$
\left|f^{n+1}\right|_{\infty}<\left(1+C_{0} \Delta t\right)\left|f^{n}\right|_{\infty},
$$

thus

$$
\left|f^{L}\right|_{\infty}<\left(1+C_{0} \Delta t\right)^{L}\left|f^{0}\right|_{\infty}<e^{C_{0} T}\left|f^{0}\right|_{\infty} .
$$

This is the $l^{\infty}$-stability property of this scheme.

\section{The schemes in higher dimensions}

Our 1D schemes can be easily extended to a higher dimension using a dimensionby-dimension approach. For example, consider the 2D Liouville equation

$$
f_{t}+\xi f_{x}+\eta f_{y}-V_{x} f_{\xi}-V_{y} f_{\eta}=0 .
$$

We employ a uniform mesh with grid points at $x_{i+\frac{1}{2}}, y_{j+\frac{1}{2}}, \xi_{k+\frac{1}{2}}, \eta_{l+\frac{1}{2}}$ in each direction. The cells are centered at $\left(x_{i}, y_{j}, \xi_{k}, \eta_{l}\right)$ with $x_{i}=\frac{1}{2}\left(x_{i+\frac{1}{2}}+x_{i-\frac{1}{2}}\right), y_{j}=$ $\frac{1}{2}\left(y_{j+\frac{1}{2}}+y_{j-\frac{1}{2}}\right), \xi_{k}=\frac{1}{2}\left(\xi_{k+\frac{1}{2}}+\xi_{k-\frac{1}{2}}\right), \eta_{l}=\frac{1}{2}\left(\eta_{l+\frac{1}{2}}+\eta_{l-\frac{1}{2}}\right)$. The mesh size is denoted by $\Delta x=x_{i+\frac{1}{2}}-x_{i-\frac{1}{2}}, \Delta y=y_{j+\frac{1}{2}}-y_{j-\frac{1}{2}}, \Delta \xi=\xi_{k+\frac{1}{2}}^{2}-\xi_{k-\frac{1}{2}}, \Delta \eta=\eta_{l+\frac{1}{2}}-\eta_{l-\frac{1}{2}}$. We define the cell average of $f$ as

$$
f_{i j k l}=\frac{1}{\Delta x \Delta y \Delta \xi \Delta \eta} \int_{x_{i-\frac{1}{2}}}^{x_{i+\frac{1}{2}}} \int_{y_{j-\frac{1}{2}}}^{y_{j+\frac{1}{2}}} \int_{\xi_{k-\frac{1}{2}}}^{\xi_{k+\frac{1}{2}}} \int_{\eta_{l-\frac{1}{2}}}^{\eta_{l+\frac{1}{2}}} f(x, y, \xi, \eta, t) d \eta d \xi d y d x .
$$

Similarly to the 1D case, we approximate the potential by a piecewise bilinear function, and for convenience, we always provide two interface values of potential at each cell interface. When the potential is smooth at a cell interface, the two potential interface values are identical. 
The 2D Liouville equation (6.1) can be semi-discretized as

$$
\begin{aligned}
\partial_{t} f_{i j k l} & +\xi_{k} \frac{f_{i+\frac{1}{2}, j k l}^{-}-f_{i-\frac{1}{2}, j k l}^{+}}{\Delta x}+\eta_{l} \frac{f_{i, j+\frac{1}{2}, k l}^{-}-f_{i, j-\frac{1}{2}, k l}^{+}}{\Delta y} \\
& -\frac{V_{i+\frac{1}{2}, j}^{-}-V_{i-\frac{1}{2}, j}^{+}}{\Delta x} \frac{f_{i j, k+\frac{1}{2}, l}-f_{i j, k-\frac{1}{2}, l}}{\Delta \xi}-\frac{V_{i, j+\frac{1}{2}}^{-}-V_{i, j-\frac{1}{2}}^{+}}{\Delta y} \frac{f_{i j k, l+\frac{1}{2}}-f_{i j k, l-\frac{1}{2}}}{\Delta \eta}=0,
\end{aligned}
$$

where the interface values $f_{i j, k+\frac{1}{2}, l}, f_{i j k, l+\frac{1}{2}}$ are provided by the upwind approximation, and the split interface values $f_{i+\frac{1}{2}, j k l}^{-}, f_{i-\frac{1}{2}, j k l}^{+}, f_{i, j+\frac{1}{2}, k l}^{-}, f_{i, j-\frac{1}{2}, k l}^{+}$can be obtained using essentially the same algorithm described in subsection 2.3 or 2.5 for the $1 \mathrm{D}$ case. Since the gradient of the potential at its smooth points is bounded, this scheme, similar to the $1 \mathrm{D}$ scheme, is also subject to a hyperbolic CFL condition under which the scheme is positive, and Hamiltonian preserving (if the discontinuity of $V$ aligns with the grids).

\section{Discontinuous solutions and numerical accuracy}

When the solution of the Liouville equation is smooth, the formally second order shock capturing finite difference scheme will produce second order numerical approximations. Consequently the physical observables obtained by evaluating the numerical $\delta$-integral concentrated on these numerical solutions, such as those in (1.8)-(1.9), should generally be of first order. However, when the potential $V$ is discontinuous, the solution of the Liouville equation, even with smooth initial data, may produce discontinuities at the downstream part of the potential discontinuity. These discontinuities influence the accuracy of the numerical $\delta$-integral through which the desired physical observables are obtained.

7.1. Discontinuities produced in the downstream part. For the Liouville equation with a discontinuous potential, if the initial data is smooth, the level set function exhibits discontinuities in the downstream side of the potential discontinuity.

We use a 1D example to illustrate this. Let $\phi$ be the level set function that solves the 1d Liouville equation with the potential given by

$$
V(x)=\left\{\begin{array}{lc}
A, & x<-b ; \\
-\frac{A x}{b}, & -b<x<0 ; \\
0, & x>0,
\end{array}\right.
$$

with $A, b$ positive. Let the initial velocity profile be a constant velocity $\xi_{0}>0$ and the initial density is denoted by $\rho_{0}(x)$. The initial value of the level set function is

$$
\phi(x, \xi, 0)=\xi-\xi_{0} .
$$

We consider the solution at $t=T$. In this example the potential is continuous, and the initial level set function is continuous, so this level set function should still be continuous at $t=T$. Now look at the set

$$
S_{1}=\left\{\left(x, \xi_{0}\right) \mid-b<x<0\right\},
$$

which is part of the initial zero level set. The bicharacteristic of the Liouville equation (2.1) is

$$
\begin{cases}\frac{d x}{d t}=\xi, & x(0)=x_{0} \\ \frac{d \xi}{d t}=-V^{\prime}(x), & \xi(0)=\xi_{0} .\end{cases}
$$


Denote the solution of (7.2) by

$$
x=x\left(x_{0}, \xi_{0}, t\right), \quad \xi=\xi\left(x_{0}, \xi_{0}, t\right),
$$

then define the set

$$
S_{2}=\left\{(x, \xi) \mid x=x\left(x_{0}, \xi_{0}, T\right), \xi=\xi\left(x_{0}, \xi_{0}, T\right),\left(x_{0}, \xi_{0}\right) \in S_{1}\right\},
$$

which is a subset of the zero level set of $\phi(x, \xi, T)$.

Select an element $\left(x_{1}, \xi_{1}\right) \in S_{2}$. We now want to evaluate $\phi_{\xi}\left(x_{1}, \xi_{1}, T\right)$. Assume $\left(x_{1}, \xi_{1}\right)=\left(x\left(-c, \xi_{0}, T\right), \xi\left(-c, \xi_{0}, T\right)\right)$. Consider the case that $T$ is large enough so that $\left(x_{1}, \xi_{1}\right)$ is a downstream point. Then the relation between $x_{1}$ and $c$ is

$$
x_{1}(c)=T \sqrt{\xi_{0}^{2}+\frac{2 A c}{b}}-\frac{b}{A}\left(\xi_{0}^{2}+\frac{2 A c}{b}\right) .
$$

The density at $x=x_{1}$ at time $T$ is the inverse of the Jacobian of $x(c)$ multiplied by the initial density

$$
\rho\left(x_{1}, T\right)=\frac{\rho_{0}(-c)}{\left|\frac{d x_{1}(c)}{d c}\right|}=\frac{b \rho_{0}(-c)}{\left|\frac{T A}{\sqrt{\xi_{0}^{2}+\frac{2 A c}{b}}}-2 b\right|} .
$$

On the other hand, it is known

$$
\rho\left(x_{1}, T\right)=\rho_{0}(-c) \int_{\xi_{1}-\epsilon}^{\xi_{1}+\epsilon} \delta\left(\phi\left(x_{1}, \xi, T\right)\right) d \xi=\frac{\rho_{0}(-c)}{\left|\phi_{\xi}\left(x_{1}, \xi_{1}, T\right)\right|} .
$$

So we have

$$
\left|\phi_{\xi}\left(x_{1}, \xi_{1}, T\right)\right|=\left|\frac{t A}{\sqrt{\xi_{0}^{2}+\frac{2 A c}{b}}}-2 b\right| b^{-1}
$$

If we take the limit $b \rightarrow 0$, we know $\left|\phi_{\xi}\left(x_{1}, \xi_{1}, T\right)\right| \rightarrow \infty$.

Moreover, the time needed for $\left(x_{1}, \xi_{1}\right)$ to be in the downstream domain, which is the time for the point $\left(-c, \xi_{0}\right)$ to reach $x=0$ along its bi-characteristic, should be $T(c)=\frac{b}{A} \sqrt{\xi_{0}^{2}+\frac{2 A c}{b}}$. Notice $T(c) \rightarrow 0$ as $b \rightarrow 0$, we know in this example when taking limit to the discontinuous potential, the level set function should contain discontinuities in the downstream domain.

7.2. Influence of discontinuities on the accuracy of the numerical evaluation of moments. In the previous subsection, we showed that the discontinuities inevitably emerge in the downstream part of the potential discontinuity. As is well known, the $l^{1}$-convergence rate for finite difference schemes to compute a discontinuous solution of a linear equation is at most halfth order [16], [29]. Here we show that a halfth order error is also introduced when evaluating the moments (1.8) (1.9) based on the discontinuous part of the solution.

We use the 1D linear advection equation with the Riemann initial data to illustrate this. Consider equation

$$
u_{t}+a u_{x}=0
$$


with initial data

$$
u(x, 0)= \begin{cases}1, & x<0 \\ -1, & x>0 .\end{cases}
$$

Since the finite difference solution is closer to the solution of the modified equation

$$
u_{t}+a u_{x}=D u_{x x}
$$

than the exact solution of the original linear advection equation (7.3), we check the accuracy of the numerical moments based on the solution of the modified equation (7.5).

If the upwind scheme is used then $D=\frac{a}{2} \Delta x\left(1-a \lambda_{x}^{t}\right)[17,28]$. Thus $D \sim \Delta x$.

The exact solution of the equation (7.5) with initial data (7.4) is

$$
\widetilde{u}(x, t)=-\frac{2}{\sqrt{\pi}} \int_{0}^{\frac{x-a t}{\sqrt{4 D} t}} e^{-z^{2}} d z .
$$

The exact solution of (7.3) with initial data (7.4) at $t$ is

$$
u(x, t)= \begin{cases}1, & x<a t \\ -1, & x>a t .\end{cases}
$$

which has the property

$$
\int \delta(u(x, t)) d x=0 .
$$

When evaluating the $\delta$-integration concentrated on (7.6), one gets

$$
\int \delta(\widetilde{u}(x, t)) d x=\frac{1}{\left|\widetilde{u}^{\prime}(a t, t)\right|}=\left.\frac{\sqrt{4 \pi D t}}{e^{-\left(\frac{x-a t}{\sqrt{4 D}}\right)^{2}}}\right|_{x=a t}=\sqrt{4 \pi D t} .
$$

Since $D \sim \Delta x$, the accuracy of numerical $\delta$-integral based on (7.6) is only halfth order. This implies that the evaluation of $\delta$-integration in (1.8)(1.9) based on the finite difference solution for (7.3) is also only halfth order.

\section{Numerical examples}

In this section we present three numerical examples to show the performance and accuracy of the proposed methods. In the numerical computations the second order TVD Runge-Kutta time discretization [26] is used. The exact solutions are constructed by the method of characteristics, taking into account the behavior of the classical particles described in Section 2.

Example 8.1. A 1D problem with an exact $L^{\infty}$-solution. Consider the 1D Liouville equation

$$
f_{t}+\xi f_{x}-V_{x} f_{\xi}=0
$$

with a discontinuous potential given by

$$
V(x)= \begin{cases}0.2, & x<0 \\ 0, & x>0 .\end{cases}
$$



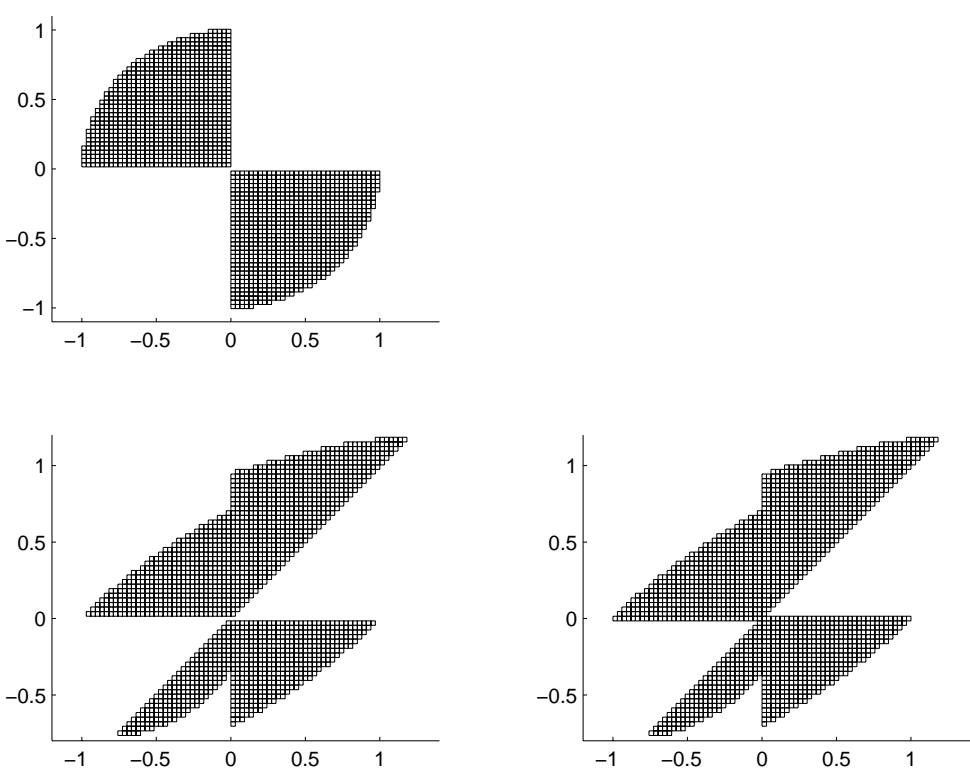

FIG. 8.1. Example 8.1, solution in the phase space. Upper: non-zero part of the initial data; lower left: non-zero part of exact solution $f(x, \xi, 1)$; lower right: the part of numerical solution $f(x, \xi, 1)>0.5$ computed by the $100 \times 101$ mesh. The horizontal axis is the position, the vertical axis is the velocity.

The initial data is given by

$$
f(x, \xi, 0)= \begin{cases}1, & x \leq 0, \xi>0, \sqrt{x^{2}+\xi^{2}}<1 ; \\ 1, & x \geq 0, \xi<0, \sqrt{x^{2}+\xi^{2}}<1 ; \\ 0, & \text { otherwise }\end{cases}
$$

as shown in the upper part of Figure 8.1 which depicts the non-zero part of $f(x, \xi, 0)$. The exact solution at $t=1$ is given by

$$
f(x, \xi, 1)= \begin{cases}1, & x \geq 0, \quad \xi<\sqrt{0.4}, \quad \xi>x ; \\ 1, & x \geq 0, \quad \xi<0, \quad x<1, \quad \xi>\frac{x-\sqrt{2-x^{2}}}{2} ; \\ 1, & x \leq 0, \quad \xi<x, \quad \xi>-\sqrt{0.6}, \quad x<\left(1-\frac{\sqrt{0.6-\xi^{2}}}{\sqrt{\xi^{2}+0.4}}\right) \xi \\ 1, & x \leq 0, \quad \xi>0, \quad x>-1, \quad \xi<\frac{x+\sqrt{2-x^{2}}}{2} \\ 1, & x \geq 0, \quad \xi>\sqrt{0.4}, \quad \xi>x, \quad \xi<\sqrt{1.4}, \quad x>\left(1-\frac{\sqrt{1.4-\xi^{2}}}{\sqrt{\xi^{2}-0.4}}\right) \xi \\ 0, & \text { otherwise },\end{cases}
$$

as shown in the lower left in Figure 8.1.

The numerical solution computed with a $100 \times 101$ mesh on the domain $[-1.5,1.5] \times[-1.5,1.5]$ using Scheme $\mathrm{I}$ is shown in the lower right in Figure 8.1. It shows a good agreement with the exact solution. 
Table 8.1 compares the $l^{1}$-error of the numerical solutions computed using $50 \times 51$, $100 \times 101$ and $200 \times 201$ meshes respectively. From these data, the convergence rate of the numerical solution in the $l^{1}$-norm is about 0.66 for both Scheme I and Scheme II. This agrees with our study in section 8, and the well established theory [16], [29], that the $l^{1}$-error by a finite difference scheme for a discontinuous solution of linear equation is at most halfth order.

Table 8.1 Example 8.1, $l^{1}$ error of the numerical solutions with different meshes

\begin{tabular}{cccc}
\hline mesh & $50 \times 51$ & $100 \times 101$ & $200 \times 201$ \\
Scheme I & 0.245192 & 0.155871 & 0.093817 \\
Scheme II & 0.246248 & 0.156963 & 0.094275 \\
\hline
\end{tabular}

Example 8.2. Computing the physical observables of a $1 \mathrm{D}$ problem with measure-valued solution. As mentioned in the Introduction, such problems arise in the computation of the semiclassical limit of the Schrödinger equation. Consider the same problem as in example 8.1, with the initial data

$$
f(x, \xi, 0)=\delta(\xi-w(x))
$$

where

$$
w(x)=\left\{\begin{array}{lr}
0.9, & x \leq-2 ; \\
0.9-\frac{0.9}{4}(x+2)^{2}, & -2<x \leq 0 \\
-0.9+\frac{0.9}{4}(x-2)^{2} ; & 0<x<2 . \\
-0.9, & x \geq 2 .
\end{array}\right.
$$

Figure 8.2 plots $w(x)$ with the dashed line.

In this example we are interested in the approximation of the moments, such as the density

$$
\rho(x, t)=\int f(x, \xi, t) d \xi
$$

and the averaged velocity

$$
u(x, t)=\frac{\int f(x, \xi, t) \xi d \xi}{\int f(x, \xi, t) d \xi}
$$

These quantities are computed by the decomposition techniques described in the Introduction. We first solve the level set function $\phi$ and the modified density function $\psi$ which satisfy the Liouville equation (8.1) with initial data $\xi-w(x)$ and 1 respectively. Then the desired physical observables $\rho$ and $u$ are computed from the numerical singular integrals (1.8), (1.9), which are computed by approximating the delta function in the integrand by a discrete delta function

$$
\delta_{\omega}(x)= \begin{cases}\frac{1}{2 \omega}\left(1+\cos \left(\frac{|\pi x|}{\omega}\right)\right), & \left|\frac{x}{\omega}\right| \leq 1 \\ 0, & \left|\frac{x}{\omega}\right|>1\end{cases}
$$




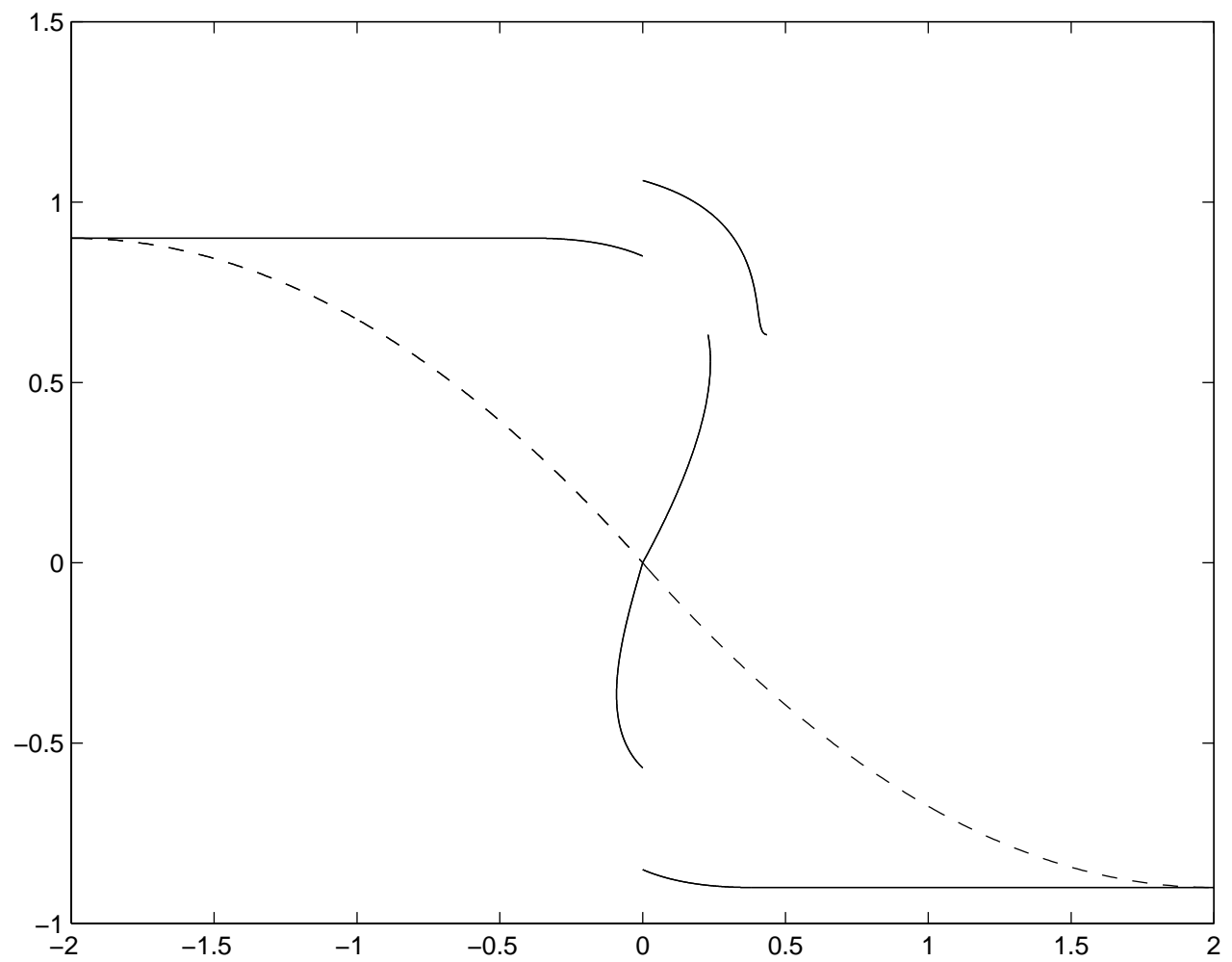

FIG. 8.2. Example 8.2, velocity. Dashed line: $w(x)$; Solid line: multivalued velocity at $t=1.8$. The horizontal axis is the position, the vertical axis is the velocity.

and then evaluating the integral on a uniform mesh $[30,5]$. The $\omega$ in $(8.6)$ is half of the support size of the discrete delta function. In our computation we choose

$$
w=\max \left(\left|\psi_{v}\right|, 1\right) h,
$$

where $\left|\psi_{v}\right|$ denotes the Jacobian of $\Psi=\left(\psi_{j}\right)$ with respect to $\mathbf{v}$ :

$$
\left|\partial \Psi / \partial\left(v_{1}, \cdots, v_{d}\right)\right|,
$$

and is approximated by the central differencing. In example $8.2 d=1$ and in example $8.3 d=2$.

The exact velocity profile and the corresponding density at $t=1.8$ are given in the Appendix. Figure 8.2 shows the exact multivalued velocity.

Figures 8.3 shows the calculated density $\rho(x, t)$ and averaged velocity $u(x, t)$ with different meshes using Scheme I together with the exact solutions. Note in the velocity the halfth order error produced around the discontinuity travels to the right and stops at around $x=1.2$. There are also such errors produced in density, but they can not be observed in the figure since these errors are small compared with the maximum density value.

Table 8.2 compares the $l^{1}$-error of the numerical densities $\rho(x, t)$ computed with $200 \times 161,400 \times 321$ and $800 \times 641$ meshes on the domain $[-2,2] \times[-1.6,1.6]$. We 

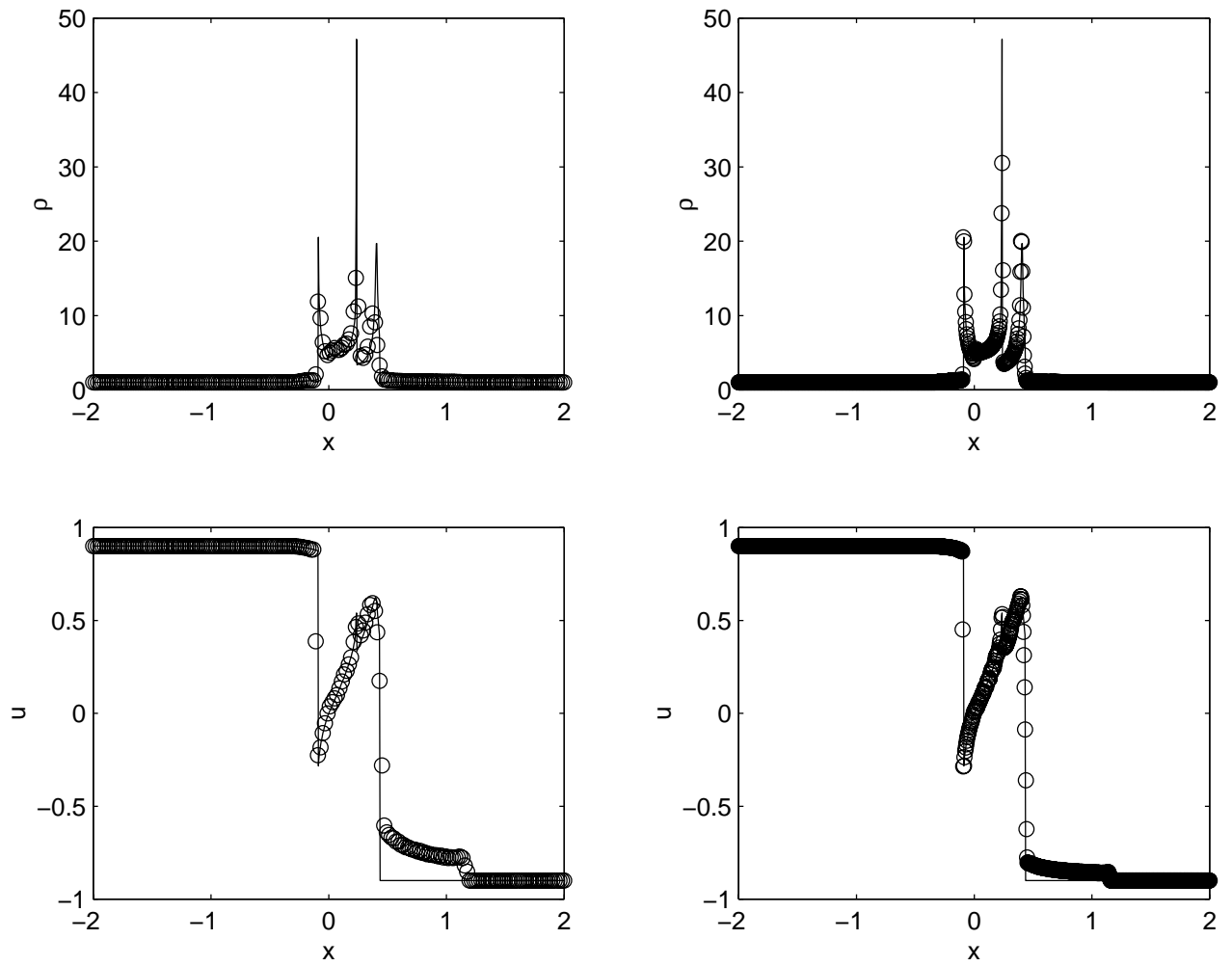

FIG. 8.3. Example 8.2, density $\rho(x, t)$ and averaged velocity $u(x, t)$ at $t=1.8$. Solid line: the exact solutions; 'o': the numerical solutions. Upper: density; lower, the averaged velocity; left: $200 \times 161$ mesh; right: $800 \times 641$ mesh.

notice that Scheme II has slightly larger errors than Scheme I. This is understandable because in order to maintain the $l^{1}$-contracting property, Scheme II may use more cell values to perform the numerical interpolation in evaluating the split fluxes.

Table $8.2 \quad l^{1}$ error of the numerical density $\rho(x, t)$ with different meshes

\begin{tabular}{cccc}
\hline mesh & $200 \times 161$ & $400 \times 321$ & $800 \times 641$ \\
Scheme I & 1.691542 & 0.967246 & 0.670656 \\
Scheme II & 1.694563 & 0.992385 & 0.679215 \\
\hline
\end{tabular}

Table 8.3 compares the $l^{1}$-error of the numerical averaged velocities $u$ computed with $200 \times 161,400 \times 321$ and $800 \times 641$ meshes on the domain $[-2,2] \times[-1.6,1.6]$. It shows the halfth order convergence.

Example 8.3. Computing the physical observables of a 2D problem with a measure-valued solution. Consider the $2 \mathrm{D}$ Liouville equation

$$
f_{t}+\xi f_{x}+\eta f_{y}-V_{x} f_{\xi}-V_{y} f_{\eta}=0
$$


Table 8.3 $\quad l^{1}$ error of the numerical averaged velocity $\rho$ on different meshes

\begin{tabular}{cccc}
\hline mesh & $200 \times 161$ & $400 \times 321$ & $800 \times 641$ \\
Scheme I & 0.170247 & 0.116522 & 0.073458 \\
Scheme II & 0.170900 & 0.128646 & 0.081642 \\
\hline
\end{tabular}

with a discontinuous potential given by

$$
V(x, y)= \begin{cases}0.1, & x>0, y>0, \\ 0, & \text { else }\end{cases}
$$

and the delta-function initial data

$$
f(x, y, \xi, \eta, 0)=\rho(x, y, 0) \delta(\xi-p(x, y)) \delta(\eta-q(x, y)),
$$

where

$$
\begin{aligned}
\rho(x, y, 0) & = \begin{cases}0, & x>-0.1, y>-0.1 \\
1, & \text { else }\end{cases} \\
p(x, y)=q(x, y) & = \begin{cases}0.4, & x>0, y>0 \\
0.6, & \text { else }\end{cases}
\end{aligned}
$$

In this example we are interested in the computation of numerical density which is the zeroth moment of this delta-type solution

$$
\rho(x, y, t)=\iint f(x, y, \xi, \eta, t) d \xi d \eta .
$$

The computational domain is chosen to be $[x, y, \xi, \eta] \in[-0.2,0.2] \times[-0.2,0.2] \times$ $[0.3,0.9] \times[0.3,0.9]$.

The exact density at $t=0.4$ is

$$
\rho(x, y, 0.4)= \begin{cases}1, & x<0 \text { or } y<0 \\ 1.5, & 0 \leq x \leq 14 / 150, y \geq \frac{3 x}{2} \\ 1.5, & 0 \leq y \leq 14 / 150, y \leq \frac{2 x}{3} \\ 0, & \text { otherwise }\end{cases}
$$

as shown in the upper left part in Figure 8.4.

The other parts in Figure 8.4 show respectively the calculated density $\rho$ with $14^{4}$, $26^{4}$ and $50^{4}$ meshes in the phase space using Scheme I in space.

Table 8.4 compares the $l^{1}$ errors on $[0,0.2] \times[0,0.2]$ of numerical densities computed with $14^{4}, 26^{4}$ and $50^{4}$ meshes in phase space. Again the error of Scheme II is larger than Scheme I. The convergence order is about $1 / 2$.

\section{Conclusion}

In this paper, we introduced two classes of Hamiltonian-preserving schemes for the Liouville equation with discontinuous potentials. By using the constant Hamiltonian across the potential barrier, we introduced a selection criterion for a unique, 
Table $8.4 \quad l^{1}$ error of numerical densities on $[0,0.2] \times[0,0.2]$ using different meshes

\begin{tabular}{cccc}
\hline mesh & $14^{4}$ & $26^{4}$ & $50^{4}$ \\
Scheme I & 0.01851 & 0.01417 & 0.01029 \\
Scheme II & 0.01864 & 0.01527 & 0.01257 \\
\hline
\end{tabular}
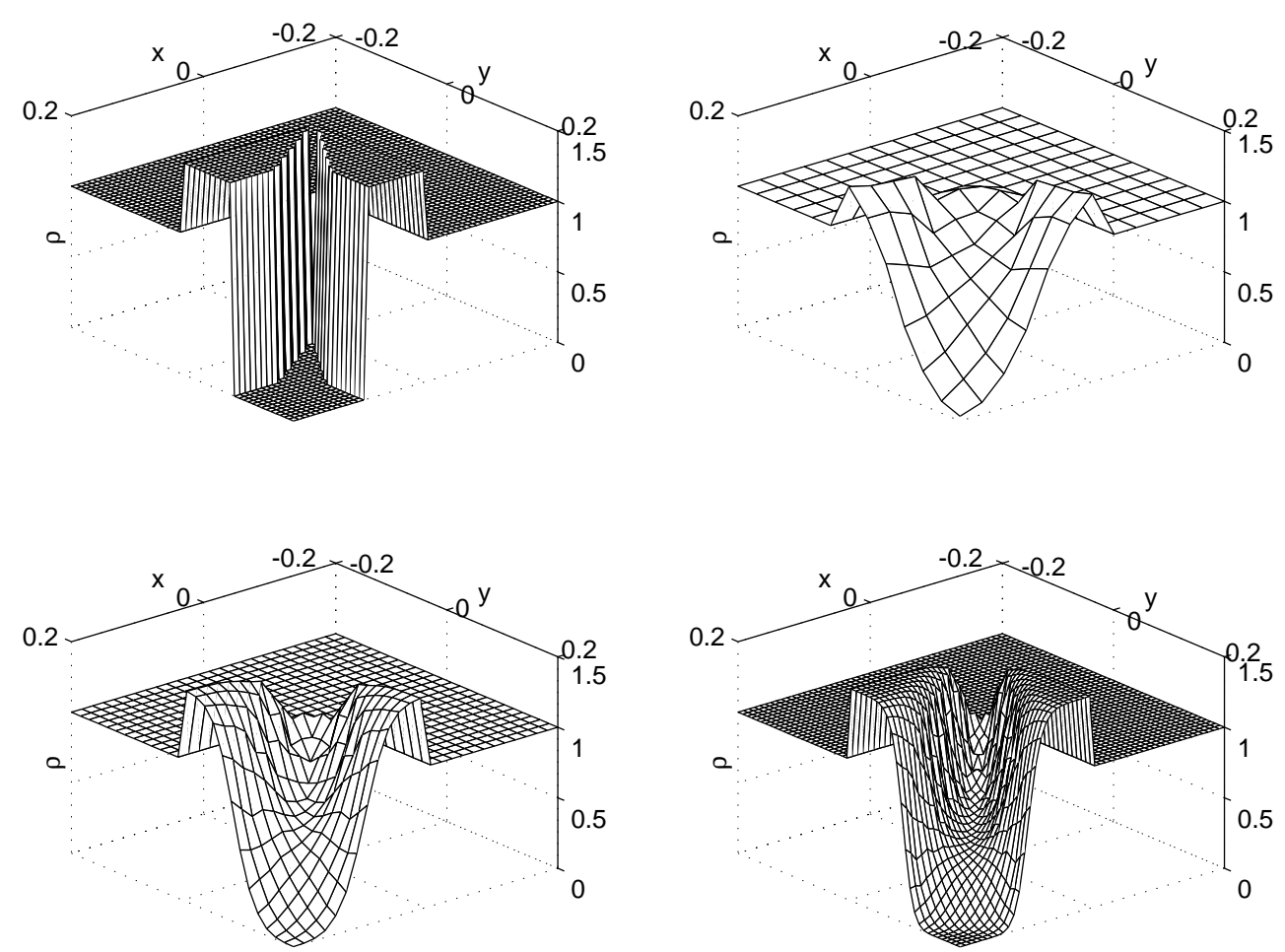

FIG. 8.4. Example 8.3, density at $t=0.4$ in space. upper left: the exact solution; Upper right: the numerical solution using $14^{4}$ mesh; lower left: the numerical solution using $26^{4}$ mesh; lower right: the numerical solution using $50^{4}$ mesh.

physically relevant solution to the underlying linear hyperbolic equation with singular coefficients. These schemes have a hyperbolic CFL condition, which is a significant improvement over a conventional discretization. We established positivity, and stability theory in both $l^{1}$ and $l^{\infty}$ norms, of these discretizations, and conducted numerical experiments to study the numerical accuracy.

This idea has also recently been extended to the Liouville equation arising from the geometrical optics limit of the linear wave equation with a discontinuous local wave speed [14], and with reflections and transmissions [15]. In addition, the same idea can also be extended to problems with external fields, such as the electrical or electromagnetic fields. There Vlasov-Poisson or Vlasov-Maxwell systems arise. Currently we are exploring the Hamiltonian-preserving schemes in these more general applications, as well as the case of a curved interface. 


\section{Appendix.}

This Appendix gives the exact velocity profile and density at $t=1.8$ for the problem in Example 8.2.

Set $t=1.8, X=-2+\sqrt{\frac{4}{0.9}(0.9-\sqrt{0.4})}+\sqrt{0.4} t$.

- In domain $-2<x<-2+0.9 t$ or $2-0.9 t<x<2$, the velocity is single phased given by

$$
u(x)= \begin{cases}0.9, & -2<x<-2+0.9 t ; \\ -0.9, & 2-0.9 t<x<2,\end{cases}
$$

the corresponding density is the constant 1.

- In domain $-2+0.9 t<x<-0.09150169603022$, the velocity is single phased given by

$$
u(x)=0.9-\frac{0.9}{4}\left(\frac{2}{0.9 t}-\sqrt{-\frac{4 x}{0.9 t}+\left(2-\frac{2}{0.9 t}\right)^{2}}\right)^{2}
$$

the corresponding density is given by

$$
\rho(x)=\frac{2}{0.9 t \sqrt{-\frac{4 x}{0.9 t}+\left(2-\frac{2}{0.9 t}\right)^{2}}} .
$$

- In domain $-0.09150169603022<x<0$, the velocity has three phases.

$$
u_{1}(x)=0.9-\frac{0.9}{4}\left(\frac{2}{0.9 t}-\sqrt{-\frac{4 x}{0.9 t}+\left(2-\frac{2}{0.9 t}\right)^{2}}\right)^{2},
$$

while $u_{2}, u_{3}$ both satisfy the expression

$$
\begin{array}{r}
-\frac{0.9 t}{4}\left(-\left(2-\frac{2}{0.9 t}\right)^{2}+\left(-\sqrt{\frac{4}{0.9}\left(0.9-\sqrt{u_{2}^{2}+0.4}\right)}+2 / 0.9 / t\right)^{2}\right) \\
\frac{u_{2}}{\sqrt{u_{2}^{2}+0.4}}=x
\end{array}
$$

and

$-0.36444353343385<u_{2}<0,-0.56860919537261<u_{3}<-0.36444353343385$.

The first branch of the density is

$$
\rho_{1}(x)=\frac{2}{0.9 t \sqrt{-\frac{4 x}{0.9 t}+\left(2-\frac{2}{0.9 t}\right)^{2}}} .
$$

To determine densities $\rho_{2}, \rho_{3}$, denote the derivative of $u_{2}(x), u_{3}(x)$ to be $u_{2}^{\prime}(x), u_{3}^{\prime}(x)$ respectively. Define the functions

$$
\begin{aligned}
& W(w)=-\sqrt{w^{2}+0.4}, \\
& X(W)=2-\sqrt{\frac{4}{0.9}(0.9+W),} \\
& Y(X)=\frac{0.9}{2}(X-2),
\end{aligned}
$$


then

$$
\begin{aligned}
& \rho_{2}(x)=\left|\frac{u_{2}(x) u_{2}^{\prime}(x)}{W\left(u_{2}(x)\right) Y\left(X\left(W\left(u_{2}(x)\right)\right)\right)}\right|, \\
& \rho_{3}(x)=\left|\frac{u_{3}(x) u_{3}^{\prime}(x)}{W\left(u_{3}(x)\right) Y\left(X\left(W\left(u_{3}(x)\right)\right)\right)}\right| .
\end{aligned}
$$

- In domain $0<x<X$, the velocity has three phases. $u_{1}$ is determined by

$$
\frac{0.9 t}{4}\left(\left(2-\frac{2}{0.9 t}\right)^{2}-\left(\sqrt{\frac{4}{0.9}\left(0.9-\sqrt{u_{1}^{2}-0.4}\right)}-\frac{2}{0.9 t}\right)^{2}\right) \frac{u_{1}}{\sqrt{u_{1}^{2}-0.4}}=x
$$

with $0.97449009909131<u_{1}<1.05986622602208$, and

$$
\begin{aligned}
& u_{2}(x)=0.9-\frac{0.9}{4}\left(-\frac{2}{0.9 t}-\sqrt{-\frac{4 x}{0.9 t}+\left(2-\frac{2}{0.9 t}\right)^{2}}\right)^{2} \\
& u_{3}(x)=-0.9+\frac{0.9}{4}\left(-\frac{2}{0.9 t}+\sqrt{\frac{4 x}{0.9 t}+\left(2-\frac{2}{0.9 t}\right)^{2}}\right)^{2} .
\end{aligned}
$$

Denote the derivative of $u_{1}(x)$ to be $u_{1}^{\prime}(x)$. Define the functions

$$
\begin{aligned}
& W(w)=\sqrt{w^{2}-0.4}, \\
& X(W)=\sqrt{\frac{4}{0.9}(0.9-W)}-2, \\
& Y(X)=-\frac{0.9}{2}(X+2),
\end{aligned}
$$

then the densities are

$$
\begin{aligned}
& \rho_{1}(x)=\left|\frac{u_{1}(x) u_{1}^{\prime}(x)}{W\left(u_{1}(x)\right) Y\left(X\left(W\left(u_{1}(x)\right)\right)\right)}\right|, \\
& \rho_{2}(x)=\frac{2}{0.9 t \sqrt{\frac{4 x}{0.9 t}+\left(2-\frac{2}{0.9 t}\right)^{2}}}, \\
& \rho_{3}(x)=\frac{2}{0.9 t \sqrt{-\frac{4 x}{0.9 t}+\left(2-\frac{2}{0.9 t}\right)^{2}}} .
\end{aligned}
$$

- In domain $X<x<-2+0.9 t+\frac{1}{0.9 t}$, the velocity has four phases. $u_{1}$ is determined by

$$
\frac{0.9 t}{4}\left(\left(2-\frac{2}{0.9 t}\right)^{2}-\left(\sqrt{\frac{4}{0.9}\left(0.9-\sqrt{u_{1}^{2}-0.4}\right)}-\frac{2}{0.9 t}\right)^{2}\right) \frac{u_{1}}{\sqrt{u_{1}^{2}-0.4}}=x
$$


with $0.96921825670040<u_{1}<0.97449009909131$, and

$$
\begin{aligned}
& u_{2}(x)=0.9-\frac{0.9}{4}\left(-\frac{2}{0.9 t}+\sqrt{-\frac{4 x}{0.9 t}+\left(2-\frac{2}{0.9 t}\right)^{2}}\right)^{2}, \\
& u_{3}(x)=0.9-\frac{0.9}{4}\left(-\frac{2}{0.9 t}-\sqrt{-\frac{4 x}{0.9 t}+\left(2-\frac{2}{0.9 t}\right)^{2}}\right)^{2}, \\
& u_{4}(x)=-0.9+\frac{0.9}{4}\left(-\frac{2}{0.9 t}+\sqrt{\frac{4 x}{0.9 t}+\left(2-\frac{2}{0.9 t}\right)^{2}}\right)^{2} .
\end{aligned}
$$

Denote the derivative of $u_{1}(x)$ to be $u_{1}^{\prime}(x)$. Define the functions

$$
\begin{aligned}
& W(w)=\sqrt{w^{2}-0.4}, \\
& X(W)=\sqrt{\frac{4}{0.9}(0.9-W)-2,} \\
& Y(X)=-\frac{0.9}{2}(X+2),
\end{aligned}
$$

then the densities are

$$
\begin{aligned}
& \rho_{1}(x)=\left|\frac{u_{1}(x) u_{1}^{\prime}(x)}{W\left(u_{1}(x)\right) Y\left(X\left(W\left(u_{1}(x)\right)\right)\right)}\right|, \\
& \rho_{2}(x)=\frac{2}{0.9 t \sqrt{-\frac{4 x}{0.9 t}+\left(2-\frac{2}{0.9 t}\right)^{2}}}, \\
& \rho_{3}(x)=\frac{2}{0.9 t \sqrt{\frac{4 x}{0.9 t}+\left(2-\frac{2}{0.9 t}\right)^{2}}} \\
& \rho_{4}(x)=\frac{2}{0.9 t \sqrt{-\frac{4 x}{0.9 t}+\left(2-\frac{2}{0.9 t}\right)^{2}}} .
\end{aligned}
$$

- In domain $-2+0.9 t+\frac{1}{0.9 t}<x<0.35899646920179$, the velocity has two phases. $u_{1}$ is determined by

$$
\frac{0.9 t}{4}\left(\left(2-\frac{2}{0.9 t}\right)^{2}-\left(\sqrt{\frac{4}{0.9}\left(0.9-\sqrt{u_{1}^{2}-0.4}\right)}-\frac{2}{0.9 t}\right)^{2}\right) \frac{u_{1}}{\sqrt{u_{1}^{2}-0.4}}=x,
$$

with $0.63245703734354<u_{1}<0.96921825670040$, and

$$
u_{2}(x)=-0.9+\frac{0.9}{4}\left(-\frac{2}{0.9 t}+\sqrt{\frac{4 x}{0.9 t}+\left(2-\frac{2}{0.9 t}\right)^{2}}\right)^{2} .
$$

Denote the derivative of $u_{1}(x)$ to be $u_{1}^{\prime}(x)$. Define the functions

$$
\begin{aligned}
& W(w)=\sqrt{w^{2}-0.4}, \\
& X(W)=\sqrt{\frac{4}{0.9}(0.9-W)-2,} \\
& Y(X)=-\frac{0.9}{2}(X+2),
\end{aligned}
$$


then the densities is given by

$$
\begin{aligned}
& \rho_{1}(x)=\left|\frac{u_{1}(x) u_{1}^{\prime}(x)}{W\left(u_{1}(x)\right) Y\left(X\left(W\left(u_{1}(x)\right)\right)\right)}\right| . \\
& \rho_{2}(x)=\frac{2}{0.9 t \sqrt{-\frac{4 x}{0.9 t}+\left(2-\frac{2}{0.9 t}\right)^{2}}} .
\end{aligned}
$$

- In domain $0.35899646920179<x<2-0.9 t$, the velocity is single phased given by

$$
u(x)=-0.9+\frac{0.9}{4}\left(-\frac{2}{0.9 t}+\sqrt{\frac{4 x}{0.9 t}+\left(2-\frac{2}{0.9 t}\right)^{2}}\right)^{2}
$$

the corresponding density is given by

$$
\rho(x)=\frac{2}{0.9 t \sqrt{\frac{4 x}{0.9 t}+\left(2-\frac{2}{0.9 t}\right)^{2}}} .
$$

\section{REFERENCES}

[1] G. Bal, J. B. Keller, G. Papanicolaou and L. Ryzhik, Transport theory for acoustic waves with reflection and transmission at interfaces, Wave Motion 30, 303-327, 1999.

[2] F. Bouchut and F. James, One-dimensional transport equations with discontinuous coefficients, Nonlinear Analysis, Theory, Methods and Applications 32, 891-933, 1998.

[3] L. T. Cheng, H. L. Liu and S. Osher, Computational high-frequency wave propagation using the level set method, with applications to the semi-classical limit of Schrodinger equations, Comm. Math. Sci. 1, 593-621, 2003.

[4] I. Capuzzo Dolcetta and B. Perthame, On some analogy between different approaches to first order PDEs with non-smooth coefficients, Adv. Math. Sci. Appl. 6, 689-703, 1996.

[5] B. Engquist, A. K. Tornberg and R. Tsai, Discretization of dirac delta functions in level set methods, J. Comp. Phys. 207, 28-51, 2005.

[6] L. Gosse, Multiphase semiclassical approximation of an electron in a one-dimensional crystalline lattice II. Impurities, confinement and Bloch oscillations, J. Comp. Phys. 201, 344-375, 2004.

[7] L. Gosse and F. James, Numerical approximations of one-dimensional linear conservation equations with discontinuous coefficients, Math. Comp. 69, 987-1015, 2000.

[8] L. Gosse, S. Jin and X. T. Li, On two moment systems for computing multiphase semiclassical limits of the schrodinger equation, Math. Model Methods Appl. Sci. 13, 1689-1723, 2003.

[9] P. Gérard, P. A. Markowich, N. J. Mauser and F. Poupaud, Homogenization limits and Wigner transforms, Comm. Pure Appl. Math. 50, 321-377, 1997.

[10] S. Jin and X. T. Li, Multi-phase computations of the semiclassical limit of the Schrödinger equation and related problems: Whitham vs. Wigner, Physica D 182, 46-85, 2003.

[11] S. Jin, H. L. Liu, S. Osher and R. Tsai, Computing multivalued physical observables for the semiclassical limit of the Schrodinger equation, J. Comp. Phys. 205, 222-241, 2005.

[12] S. Jin and S. Osher, A level set method for the computation of multivalued solutions to quasilinear hyperbolic PDE's and Hamilton-Jacobi equations, Comm. Math. Sci. 1, 575-591, 2003.

[13] S. Jin and X. Wen, The $l^{1}$-stability of a Hamiltonian-preserving scheme for the Liouville equation with discontinuous potentials, preprint.

[14] S. Jin and X. Wen, Hamiltonian-preserving schemes for the Liouville equation of geometrical optics with discontinuous local wave speeds, submitted.

[15] S. Jin and X. Wen, Hamiltonian-preserving schemes for the Liouville equation of geometrical optics with transmissions and reflections, submitted.

[16] N. N. Kuznetsov, On stable methods for solving non-linear first order partial differential equations in the class of discontinuous functions, Topics in Numerical Analysis III (Proc. Roy. Irish Acad. Conf.)(J. J. H. Miller, ad.), Academic Press, London, 183-197, 1977. 
[17] R.J. LeVeque, Numerical Methods for Conservation Laws, Birkhauser-Verlag, Basel, 1990.

[18] P. L. Lions and T. Paul, Sur les measures de Wigner, Revista. Mat. Iberoamericana 9, 1993, 553-618.

[19] L. Miller, Refraction of high frequency waves density by sharp interfaces and semiclassical measures at the boundary, J. Math. Pures Appl. IX 79, 227-269, 2000.

[20] B. Perthame and C. W. Shu, On positivity preserving finite volume schemes for Euler equations, Numer. Math. 73, 119-130, 1996.

[21] B. Perthame and C. Simeoni, A kinetic scheme for the Saint-Venant system with a source term, CALCOLO 38, 201-231, 2001

[22] G. Petrova and B. Popov, Linear transport equations with discontinuous coefficients, J. Math. Anal. Appl. 260, 307-324, 2001.

[23] F. Poupaud and M. Rascle, Measure solutions to the linear multidimensional transport equation with non-smooth coefficients, Comm. PDEs 22, 337-358, 1997.

[24] L. Ryzhik, G. Papanicolaou and J. Keller, Transport equations for elastic and other waves in random media, Wave Motion 24, 327-370, 1996.

[25] L. Ryzhik, G. Papanicolaou and J. Keller, Transport equations for waves in a half space, Comm. PDE's 22, 1869-1910, 1997.

[26] C. W. Shu and S. Osher, Efficient implementation of essentially non-oscillatory shock capturing scheme, J. Comput. Phys. 77, 439-471, 1988.

[27] C. Sparber, P. Markowich and N. Mauser, Multivalued geometrical optics: Wigner vs. WKB, Asymptotic Analysis 33, 153-187, 2003

[28] P. K. Sweby, High-resolution schemes using flux limiters for hyperbolic conservation-laws, SIAM J. Num. Anal. 21, 995-1011, 1984.

[29] T. Tang and Z. H. Teng, The sharpness of Kuznetsov's $O(\sqrt{\Delta x})$ L1-error estimate for monotone difference schemes, Math. Comp. 64, 581-589, 1995.

[30] A. K. Tornberg, Multi-dimensional quadrature of singular and discontinuous functions, BIT $42,644-669,2002$ 